Commun. Fac. Sci. Univ. Ank. Ser. A1 Math. Stat.

Volume 69, Number 1, Pages 486-510(2020)

DOI: $10.31801 /$ cfsuasmas. 577438

ISSN 1303-5991 E-ISSN 2618-6470

http://communications.science.ankara.edu.tr/index.php?series=A1

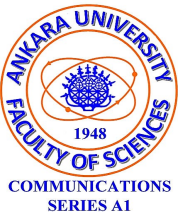

\title{
ASYMPTOTIC BEHAVIOUR OF RESONANCE EIGENVALUES OF THE SCHRÖDINGER OPERATOR WITH A MATRIX POTENTIAL
}

\author{
SEDEF KARAKILIÇ, SETENAY AKDUMAN, AND DIDEM COŞKAN
}

\begin{abstract}
We will discuss the asymptotic behaviour of the eigenvalues of a Schrödinger operator with a matrix potential defined by the Neumann boundary condition in $L_{2}^{m}(F)$, where $F$ is a $d$-dimensional rectangle and the potential is an $m \times m$ matrix with $m \geq 2, d \geq 2$, when the eigenvalues belong to the resonance domain, roughly speaking they lie near the planes of diffraction.
\end{abstract}

\section{Introduction}

In this paper, we consider the Schrödinger operator with a matrix potential $V(x)$ defined by the differential expression

$$
L \phi=-\Delta \phi+V \phi
$$

and the Neumann boundary condition

$$
\left.\frac{\partial \phi}{\partial n}\right|_{\partial F}=0
$$

in $L_{2}^{m}(F)$ where $F$ is the $d$ dimensional rectangle $F=\left[0, a_{1}\right] \times\left[0, a_{2}\right] \times \ldots \times\left[0, a_{d}\right]$, $\partial F$ is the boundary of $F, m \geqslant 2, d \geqslant 2, \frac{\partial}{\partial n}$ denotes differentiation along the outward normal of the boundary $\partial F, \Delta$ is a diagonal $m \times m$ matrix whose diagonal elements are the scalar Laplace operators $\triangle=\frac{\partial^{2}}{\partial x_{1}{ }^{2}}+\frac{\partial^{2}}{\partial x_{2}{ }^{2}}+\ldots+\frac{\partial^{2}}{\partial x_{d}{ }^{2}}, x=\left(x_{1}, x_{2}, \ldots, x_{d}\right) \in$ $R^{d}, V$ is a real valued symmetric matrix $V(x)=\left(v_{i j}(x)\right), i, j=1,2, \ldots, m, v_{i j}(x) \in$ $L_{2}(F)$, that is, $V^{T}(x)=V(x)$.

We denote the operator defined by (1)-(2) by $L(V)$, the eigenvalues and the corresponding eigenfunctions of $L(V)$ by $\Lambda_{N}$ and $\Psi_{N}$, respectively.

The eigenvalues of the operator $L(0)$ which is defined by the differential expression (1) when $V(x)=0$ and the boundary condition (2) are $|\gamma|^{2}$, and the

Received by the editors: June 16, 2019; Accepted: November 27, 2019.

2010 Mathematics Subject Classification. 47F05, 35P15.

Key words and phrases. Schrödinger operator, Neumann condition, Resonance eigenvalue, perturbation theory.

(C) 2020 Ankara University Communications Faculty of Sciences University of Ankara-Series A1 Mathematics and Statistics 
corresponding eigenspaces are $E_{\gamma}=\operatorname{span}\left\{\Phi_{\gamma, 1}(x), \Phi_{\gamma, 2}(x), \ldots, \Phi_{\gamma, m}(x)\right\}$, where

$$
\begin{aligned}
\gamma & =\left(\gamma^{1}, \gamma^{2}, \ldots, \gamma^{d}\right) \in \frac{\Gamma^{+0}}{2}, \\
\frac{\Gamma^{+0}}{2} & =\left\{\left(\frac{n_{1} \pi}{a_{1}}, \frac{n_{2} \pi}{a_{2}} \cdots, \frac{n_{d} \pi}{a_{d}}\right): n_{k} \in Z^{+} \cup\{0\}, k=1,2, \ldots, d\right\}, \\
\Phi_{\gamma, j}(x) & =\left(0, \ldots, 0, u_{\gamma}(x), 0, \ldots, 0\right), j=1,2, \ldots, m,
\end{aligned}
$$

and the non-zero component of $\Phi_{\gamma, j}(x)$ is $u_{\gamma}(x)=\cos \frac{n_{1} \pi}{a_{1}} x_{1} \cos \frac{n_{2} \pi}{a_{2}} x_{2} \cdots \cos \frac{n_{d} \pi}{a_{d}} x_{d}$, which stands in the $j$ th component. In particular, $u_{0}(x)=1$ when $\gamma=(0,0, \ldots, 0)$.

It can be easily calculated that the norm of $u_{\gamma}(x), \gamma \in \frac{\Gamma^{+0}}{2}$, in $L_{2}(F)$ is $\sqrt{\frac{\mu(F)}{\left|A_{\gamma}\right|}}$, where $\mu(F)$ is the measure of the $d$-dimensional parallelepiped $F,\left|A_{\gamma}\right|$ is the number of vectors in $A_{\gamma}=\left\{\alpha=\left(\alpha_{1}, \alpha_{2}, \ldots, \alpha_{d}\right) \in \frac{\Gamma}{2}:\left|\alpha_{k}\right|=\left|\gamma^{k}\right|, k=1,2, \ldots, d\right\}, \frac{\Gamma}{2}=$ $\left\{\left(\frac{n_{1} \pi}{a_{1}}, \frac{n_{2} \pi}{a_{2}} \cdots, \frac{n_{d} \pi}{a_{d}}\right): n_{k} \in Z, k=1,2, \ldots, d\right\}$.

From now on, $\langle.,$.$\rangle and (.,$.$) will denote the inner products in L_{2}^{m}(F)$ and $L_{2}(F)$, respectively.

Since $\left\{u_{\gamma}(x)\right\}_{\gamma \in \frac{\Gamma^{+0}}{2}}$ is a complete system in $L_{2}(F)$, for any $q(x)$ in $L_{2}(F)$ we have

$$
q(x)=\sum_{\gamma \in \frac{\Gamma^{+0}}{2}} \frac{\left|A_{\gamma}\right|}{\mu(F)}\left(q, u_{\gamma}\right) u_{\gamma}(x) .
$$

In our study, it is convenient to use the equivalent decomposition (see 9])

$$
q(x)=\sum_{\gamma \in \frac{\Gamma}{2}} q_{\gamma} u_{\gamma}(x),
$$

where $q_{\gamma}=\frac{1}{\mu(F)}\left(q(x), u_{\gamma}(x)\right)$ for the sake of simplicity. That is, the decomposition (3) and (4) are equivalent for any $d \geq 2$. Thus, according to (4), each matrix element $v_{i j}(x) \in L_{2}(F)$ of the matrix $V(x)$ can be written in its Fourier series expansion

$$
v_{i j}(x)=\sum_{\gamma \in \frac{\Gamma}{2}} v_{i j \gamma} u_{\gamma}(x),
$$

$v_{i j \gamma}=\frac{\left(v_{i j}, u_{\gamma}\right)}{\mu(F)},\left(v_{i j}, u_{\gamma}\right)=\frac{1}{\mu(F)} \int_{F} v_{i j}(x) u_{\gamma}(x) d x$ and $v_{i j 0}=\frac{1}{\mu(F)} \int_{F} v_{i j}(x) d x i, j=$ $1,2, \ldots, m$.

We assume that $l>\frac{(d+20)(d-1)}{2}+d+3$ and the Fourier coefficients $v_{i j \gamma}$ of $v_{i j}(x)$ satisfy

$$
\sum_{\gamma \in \frac{\Gamma}{2}}\left|v_{i j \gamma}\right|^{2}\left(1+|\gamma|^{2 l}\right)<\infty,
$$

for each $i, j=1,2, \ldots, m$. Let $\rho$ be a large parameter, $\rho \gg 1$ and $\alpha$ be a positive number with $0<\alpha<\frac{1}{d+20}$ then for $\Gamma\left(\rho^{\alpha}\right)=\left\{\gamma \in \frac{\Gamma}{2}: 0 \leq|\gamma|<\rho^{\alpha}\right\}$ and $p=l-d$ 
the condition (6) implies that

$$
v_{i j}(x)=\sum_{\gamma \in \Gamma\left(\rho^{\alpha}\right)} v_{i j \gamma} u_{\gamma}(x)+O\left(\rho^{-p \alpha}\right) .
$$

Here $O\left(\rho^{-p \alpha}\right)$ is a function in $L_{2}(F)$ with norm of order $\rho^{-p \alpha}$. Furthermore, by (6), we have

$$
M_{i j} \equiv \sum_{\gamma \in \frac{\Gamma}{2}}\left|v_{i j \gamma}\right|<\infty
$$

for all $i, j=1,2, \ldots, m$.

Notice that, if a function $q(x)$ is $\operatorname{sufficiently~} \operatorname{smooth}\left(q(x) \in W_{2}^{l}(F)\right)$ and the support of $\nabla q(x)=\left(\frac{\partial q}{\partial x_{1}}, \frac{\partial q}{\partial x_{2}}, \ldots, \frac{\partial q}{\partial x_{d}}\right)$ is contained in the interior of the domain $F$, then $q(x)$ satisfies condition (6) (See [7]). There is also another class of functions $q(x)$, such that $q(x) \in W_{2}^{l}(F)$,

$$
q(x)=\sum_{\gamma^{\prime} \in \Gamma} q_{\gamma^{\prime}} u_{\gamma^{\prime}}(x),
$$

which is periodic with respect to a lattice

$$
\Omega=\left\{\left(m_{1} a_{1}, m_{2} a_{2}, \ldots, m_{d} a_{d}\right): m_{k} \in \boldsymbol{Z}, k=1,2, \ldots, d\right\}
$$

and thus it also satisfies condition (6).

As in [17-22, we divide $R^{d}$ into two domains: Resonance and Non-resonance domains. In order to define these domains, let us introduce the following sets:

Let $0<\alpha<\frac{1}{d+20}, \alpha_{k}=3^{k} \alpha, k=1,2, \ldots, d-1$ and

$$
\begin{aligned}
V_{b}\left(\rho^{\alpha_{1}}\right) & \equiv\left\{x \in R^{d}:\left.|| x\right|^{2}-|x+b|^{2} \mid<\rho^{\alpha_{1}}\right\} \\
E_{1}\left(\rho^{\alpha_{1}}, p\right) & \equiv \bigcup_{b \in \Gamma\left(p \rho^{\alpha}\right)} V_{b}\left(\rho^{\alpha_{1}}\right) \\
U\left(\rho^{\alpha_{1}}, p\right) & \equiv R^{d} \backslash E_{1}\left(\rho^{\alpha_{1}}, p\right) \\
E_{k}\left(\rho^{\alpha_{k}}, p\right) & =\bigcup_{\gamma_{1}, \gamma_{2}, \ldots, \gamma_{k} \in \Gamma\left(p \rho^{\alpha}\right)}\left(\bigcap_{i=1}^{k} V_{\gamma_{i}}\left(\rho^{\alpha_{k}}\right)\right)
\end{aligned}
$$

where $b \neq 0, \gamma_{i} \neq 0, i=1,2, \ldots, k$ and the intersection $\bigcap_{i=1}^{k} V_{\gamma_{i}}\left(\rho^{\alpha_{k}}\right)$ in $E_{k}$ is taken over $\gamma_{1}, \gamma_{2}, \ldots, \gamma_{k}$ which are linearly independent vectors and the length of $\gamma_{i}$ is not greater than the length of the other vector in $\Gamma \bigcap \gamma_{i} R$. The set $U\left(\rho^{\alpha_{1}}, p\right)$ is said to be a non-resonance domain, and the eigenvalue $|\gamma|^{2}$ is called a non-resonance eigenvalue if $\gamma \in U\left(\rho^{\alpha_{1}}, p\right)$. The domains $V_{b}\left(\rho^{\alpha_{1}}\right)$, for $b \in \Gamma\left(p \rho^{\alpha}\right)$ are called resonance domains and the eigenvalue $|\gamma|^{2}$ is a resonance eigenvalue if $\gamma \in V_{b}\left(\rho^{\alpha_{1}}\right)$. 
As noted in [20]-21], the domain $V_{b}\left(\rho^{\alpha_{1}}\right) \backslash E_{2}$, called a single resonance domain, has asymptotically full measure on $V_{b}\left(\rho^{\alpha_{1}}\right)$, that is,

$$
\frac{\mu\left(\left(V_{b}\left(\rho^{\alpha_{1}}\right) \backslash E_{2}\right) \bigcap B(q)\right)}{\mu\left(V_{b}\left(\rho^{\alpha_{1}}\right) \bigcap B(q)\right)} \rightarrow 1, \text { as } \rho \rightarrow \infty,
$$

where $B(\rho)=\left\{x \in \boldsymbol{R}^{d}:|x|=\rho\right\}$, if

$$
2 \alpha_{2}-\alpha_{1}+(d+3) \alpha<1, \quad \alpha_{2}>2 \alpha_{1},
$$

hold. Since $0<\alpha<\frac{1}{d+20}$, the conditions in 9 hold.

In most cases, it is important to know the asymptotic behavior of the eigenvalues of the Schrödinger operator $L(V)$. In this paper, [3] and [8, we construct the asymptotic formulas in the high energy region for eigenvalues of the operator $L(V)$.

In [3], we obtain the asymptotic formulas of arbitrary order for the eigenvalue of $L(V)$ corresponding to the non-resonance eigenvalues $|\gamma|^{2}$ of $L(0)$ in arbitrary dimension $d \geq 2$.

In [8, we constructed the high energy asymptotics of arbitrary order for the eigenvalue of $L(V)$ corresponding to resonance eigenvalue $|\gamma|^{2}$ when $\gamma$ belongs to the special single resonance domains $V_{\delta}\left(\rho^{\alpha_{1}}\right) \backslash E_{2}$, where $\delta$ is from $\left\{e_{1}, e_{2}, \ldots, e_{d}\right\}$ and $e_{1}=\left(\frac{\pi}{a_{1}}, 0, \ldots, 0\right), \ldots, e_{d}=\left(0, \ldots, \frac{\pi}{a_{d}}\right), d \geq 2$.

In this paper, we study the case for which $|\gamma|^{2}$ is a resonance eigenvalue. More precisely, in Theorem (1) and (2) of Section(2), we assume that $\gamma \in\left(\bigcap_{i=1}^{k} V_{\gamma_{i}}\left(\rho^{\alpha_{k}}\right)\right) \backslash$ $E_{k+1}, k=1,2, \ldots, d-1$ and $\gamma \notin V_{e_{k}}\left(\rho^{\alpha_{1}}\right)$ for $k=1,2, \ldots, d$ and prove that the corresponding eigenvalue of $L(V)$ is close to the sum of the eigenvalue of the matrix $V_{0}$ and the eigenvalue of the matrix $C=C\left(\gamma, \gamma_{1}, \ldots, \gamma_{k}\right)$ (See (14)).

In Section(3), this time we assume that $\gamma \in V_{\delta}\left(\rho^{\alpha_{1}}\right) \backslash E_{2}, \delta \in \frac{T}{2} \backslash\left\{e_{1}, e_{2}, \ldots, e_{d}\right\}$, that is, $\gamma$ is in a single resonance domain and we prove the main result Theorem (7) which gives a connection between the eigenvalues of $L(V)$ corresponding to a single resonance domain and the eigenvalues of the Sturm-Liouville operators.

Note that, the case $\delta=e_{i}, i=1,2, \ldots, d$, was considered in [8], by a different but simpler method and better formulas were obtained.

\section{Asymptotic Formulas for the Eigenvalues in the Resonance DOMAIN}

We assume that $\gamma \notin V_{e_{k}}\left(\rho^{\alpha_{1}}\right)$ for $k=1,2, \ldots, d$, and $|\gamma|^{2}$ is a resonance eigenvalue of the operator $L(0)$, that is, $\gamma \in\left(\bigcap_{i=1}^{k} V_{\gamma_{i}}\left(\rho^{\alpha_{k}}\right)\right) \backslash E_{k+1}, k=1,2, \ldots, d-1$, such that $|\gamma| \sim \rho$ where $|\gamma| \sim \rho$ means that $|\gamma|$ and $\rho$ are asymptotically equal, that is, there exist $c_{1}, c_{2}$ satisfying the inequality $c_{1} \rho \leq|\gamma| \leq c_{2} \rho, c_{i}, i=1,2,3, \ldots$ 
are positive real constants which do not depend on $\rho$. To obtain the asymptotic formulas for the eigenvalues of $L(V)$ corresponding to $|\gamma|^{2}$ we use the binding formula (see $(9)$ in 3 ])

$$
\left(\Lambda_{N}-|\gamma|^{2}\right)\left\langle\Psi_{N}, \Phi_{\gamma, j}\right\rangle=\left\langle\Psi_{N}, V \Phi_{\gamma, j}\right\rangle .
$$

Now, we decompose $V(x) \Phi_{\gamma, j}(x)$ with respect to the basis $\left\{\Phi_{\gamma^{\prime}, i}(x)\right\}_{\gamma^{\prime} \in \frac{\Gamma}{2}, i=1,2, \ldots, m}$. By definition of $\Phi_{\gamma, j}(x)$, it is obvious that

$$
V(x) \Phi_{\gamma, j}(x)=\left(v_{1 j}(x) u_{\gamma}(x), \ldots, v_{m j}(x) u_{\gamma}(x)\right) .
$$

Substituting the decomposition (7) of $v_{i j}(x)$ in (11), we get

$V(x) \Phi_{\gamma, j}(x)=\left(\sum_{\gamma \prime \in \Gamma\left(\rho^{\alpha}\right)} v_{1 j \gamma^{\prime}} u_{\gamma \prime}(x) u_{\gamma}(x), \ldots, \sum_{\gamma \prime \in \Gamma\left(\rho^{\alpha}\right)} v_{m j \gamma^{\prime}} u_{\gamma^{\prime}}(x) u_{\gamma}(x)\right)+O\left(\rho^{-p \alpha}\right)$.

Since $\gamma$ does not belong to the domains $V_{e_{k}}\left(\rho^{\alpha_{1}}\right)$, for each $k=1,2, \ldots d$, we may use the following equation

$$
\sum_{\gamma \prime \in \Gamma\left(\rho^{\alpha}\right)} v_{i j \gamma^{\prime}} u_{\gamma^{\prime}}(x) u_{\gamma}(x)=\sum_{\gamma \prime \in \Gamma\left(\rho^{\alpha}\right)} v_{i j \gamma^{\prime}} u_{\gamma-\gamma^{\prime}}(x)
$$

which is proved in [9] (see equation (18) in [9]), and obtain

$$
\begin{aligned}
V(x) \Phi_{\gamma, j}(x) & =\left(\sum_{\gamma \prime \in \Gamma\left(\rho^{\alpha}\right)} v_{1 j \gamma^{\prime}} u_{\gamma-\gamma^{\prime}}(x), \ldots, \sum_{\gamma \prime \in \Gamma\left(\rho^{\alpha}\right)} v_{m j \gamma^{\prime}} u_{\gamma-\gamma^{\prime}}(x)\right)+O\left(\rho^{-p \alpha}\right) \\
& =\sum_{i=1}^{m} \sum_{\gamma \prime \in \Gamma\left(\rho^{\alpha}\right)} v_{i j \gamma^{\prime}} \Phi_{\gamma-\gamma^{\prime}, i}(x)+O\left(\rho^{-p \alpha}\right) .
\end{aligned}
$$

Substituting $(12)$ into $(10)$, we obtain

$$
\begin{aligned}
<\Psi_{N}, \Phi_{\gamma, j}> & =\frac{<\Psi_{N}, V \Phi_{\gamma, j}>}{\left(\Lambda_{N}-|\gamma|^{2}\right)} \\
& =\sum_{i=1}^{m} \sum_{\gamma / \in \Gamma\left(\rho^{\alpha}\right)} v_{i j \gamma^{\prime}} \frac{<\Psi_{N}, \Phi_{\gamma-\gamma^{\prime}, i}>}{\left(\Lambda_{N}-|\gamma|^{2}\right)}+O\left(\rho^{-p \alpha}\right)
\end{aligned}
$$

for every vector $\gamma \in \frac{\Gamma}{2}$, satisfying the condition

$$
\left.\left|\Lambda_{N}-\right| \gamma\right|^{2} \mid>\frac{1}{2} \rho^{\alpha_{1}}
$$

Letting $p_{1}=\left[\frac{p+1}{2}\right]$, that is, $p_{1}$ is the integer part of $\frac{p+1}{2}$, we define the following sets

$$
\begin{gathered}
B_{k}\left(\gamma_{1}, \gamma_{2}, \ldots, \gamma_{k}\right)=\left\{b: b=\sum_{i=1}^{k} n_{i} \gamma_{i}, n_{i} \in Z,|b|<\frac{1}{2} \rho^{\frac{1}{2} \alpha_{k+1}}\right\} \\
B_{k}(\gamma)=\gamma+B_{k}\left(\gamma_{1}, \gamma_{2}, \ldots, \gamma_{k}\right)=\left\{\gamma+b: b \in B_{k}\left(\gamma_{1}, \gamma_{2}, \ldots, \gamma_{k}\right)\right\} \\
B_{k}\left(\gamma, p_{1}\right)=B_{k}(\gamma)+\Gamma\left(p_{1} \rho^{\alpha}\right) .
\end{gathered}
$$


Let $h_{\tau}, \tau=1,2, \ldots, b_{k}$ denote the vectors of $B_{k}\left(\gamma, p_{1}\right), b_{k}$ the number of the vectors in $B_{k}\left(\gamma, p_{1}\right)$. By its definition, it can easily be obtained that $b_{k}=O\left(\rho^{\frac{d}{2} 3^{d} \alpha}\right)$, since $\alpha_{k}=3^{k} \alpha, 2 \leq k \leq d$. We define the $m b_{k} \times m b_{k}$ matrix $C=C\left(\gamma, \gamma_{1}, \ldots, \gamma_{k}\right)$ by

$$
C=\left[\begin{array}{cccc}
\left|h_{1}\right|^{2} I-V_{0} & V_{h_{1}-h_{2}} & \cdots & V_{h_{1}-h_{b_{k}}} \\
V_{h_{2}-h_{1}} & \left|h_{2}\right|^{2} I-V_{0} & \cdots & V_{h_{2}-h_{b_{k}}} \\
\vdots & & & \\
V_{h_{b_{k}}-h_{1}} & V_{h_{b_{k}}-h_{2}} & \cdots & \left|h_{b_{k}}\right|^{2} I-V_{0}
\end{array}\right],
$$

where $V_{h_{\tau}-h_{\xi}}, \tau, \xi=1,2, \ldots, b_{k}$ are the $m \times m$ matrices defined by

$$
V_{h_{\tau}-h_{\xi}}=\left[\begin{array}{cccc}
v_{11 h_{\tau}-h_{\xi}} & v_{12 h_{\tau}-h_{\xi}} & \cdots & v_{1 m h_{\tau}-h_{\xi}} \\
v_{21 h_{\tau}-h_{\xi}} & v_{22 h_{\tau}-h_{\xi}} & \cdots & v_{2 m h_{\tau}-h_{\xi}} \\
\vdots & & & \\
v_{m 1 h_{\tau}-h_{\xi}} & v_{m 2 h_{\tau}-h_{\xi}} & \cdots & v_{m m h_{\tau}-h_{\xi}}
\end{array}\right] .
$$

Writing equation 13 for all $h_{\tau} \in B_{k}\left(\gamma, p_{1}\right), \tau=1,2, \ldots, b_{k}$ and $j=1,2, \ldots, m$, we get

$$
\left(\Lambda_{N}-\left|h_{\tau}\right|^{2}\right)<\Psi_{N}, \Phi_{h_{\tau}, j}>=\sum_{i=1}^{m} \sum_{\gamma / \in \Gamma\left(\rho^{\alpha}\right)} v_{i j \gamma^{\prime}}<\Psi_{N}, \Phi_{h_{\tau}-\gamma^{\prime}, i}>+O\left(\rho^{-p \alpha}\right) .
$$

Similar system of equations for quasi-periodic boundary condition was investigated in [19], 21] and 22]. More recently, in [22], Lemma 2.2.1. states that for $\gamma \in$ $\left(\bigcap^{k} V_{\gamma_{i}}\left(\rho^{\alpha_{k}}\right)\right) \backslash E_{k+1}, h_{\tau} \in B_{k}\left(\gamma, p_{1}\right)$ and $\gamma^{\prime}, \gamma_{1}, \gamma_{2}, \ldots, \gamma_{s} \in \Gamma\left(\rho^{\alpha}\right)$, if $h_{\tau}-\gamma^{\prime} \notin$ $\stackrel{i=1}{B_{k}\left(\gamma, p_{1}\right) \text { then }}$

$$
\left.|| \gamma\right|^{2}-\left|h_{\tau}-\gamma \prime-\gamma_{1}-\ldots-\gamma_{s}\right|^{2} \mid>\frac{1}{5} \rho^{\alpha_{k+1}},
$$

for $s=0,1,2, \ldots, p_{1}-1$.

Thus, if an eigenvalue $\Lambda_{N}$ of $L(V)$ satisfies

$$
\left.\left|\Lambda_{N}-\right| \gamma\right|^{2} \mid<\frac{1}{2} \rho^{\alpha_{1}}
$$

then by (17) and $(18)$, we have

$$
\left|\Lambda_{N}-\right| h_{\tau}-\gamma \prime-\gamma_{1}-\ldots-\left.\gamma_{s}\right|^{2} \mid>\frac{1}{6} \rho^{\alpha_{k+1}} .
$$

Now, we prove that if 18 holds then

$$
O\left(\rho^{-p \alpha}\right)=\sum_{i=1}^{m} \sum_{\substack{\gamma \in \Gamma\left(\rho^{\alpha}\right) \\ h_{\tau}-\gamma \prime \notin B \\-\gamma\left(\gamma, p_{1}\right)}} v_{i j \gamma \prime}<\Psi_{N}, \Phi_{h_{\tau}-\gamma^{\prime}, i}>
$$


for any $j=1,2, \ldots, m$. Here we remark that $\gamma^{\prime} \neq 0$. If it were the case, then we would have from $h_{\tau}-\gamma^{\prime} \notin B_{k}\left(\gamma, p_{1}\right)$ that $h_{\tau} \notin B_{k}\left(\gamma, p_{1}\right)$ which is a contradiction. So, to prove (20), we argue as Theorem 2.2.2 (a) of [22]: Since $\Lambda_{N}$ satisfies the inequality 18$)$, by 19$)$ (for $s=0$ ) we have $\left|\Lambda_{N}-\right| h_{\tau}-\left.\gamma^{\prime}\right|^{2} \mid>\frac{1}{6} \rho^{\alpha_{k+1}}$. Using this, in the equation (13) instead of $\gamma$ we write $h_{\tau}-\gamma /$ to get

$$
<\Psi_{N}, \Phi_{h_{\tau}-\gamma^{\prime}, j}>=\sum_{i_{1}=1}^{m} \sum_{\gamma_{1} \in \Gamma\left(\rho^{\alpha}\right)} v_{i j \gamma_{1}} \frac{<\Psi_{N}, \Phi_{h_{\tau}-\gamma^{\prime}-\gamma_{1}, i_{1}}>}{\left(\Lambda_{N}-\left|h_{\tau}-\gamma^{\prime}\right|^{2}\right)}+O\left(\rho^{-p \alpha}\right) .
$$

Substituting this equation (21) into the right hand side of $(20)$, we obtain

$$
\begin{aligned}
& \sum_{\substack{\gamma \in \in \Gamma\left(\rho^{\alpha}\right) \\
h_{\tau}-\gamma^{\prime} \notin B_{k}\left(\gamma, p_{1}\right)}} v_{i j \gamma^{\prime}}<\Psi_{N}, \Phi_{h_{\tau}-\gamma^{\prime}, i}> \\
& \sum_{\substack{\gamma \prime \in \Gamma\left(\rho^{\alpha}\right) \\
h_{\tau}-\gamma^{\prime} \notin B_{k}\left(\gamma, p_{1}\right)}} \frac{v_{i j \gamma^{\prime}}}{\Lambda_{N}-\left|h_{\tau}-\gamma^{\prime}\right|^{2}} \sum_{i_{1}=1}^{m} \sum_{\substack{h_{1} \in \Gamma\left(\rho^{\alpha}\right) \\
h_{\tau}-\gamma \notin \notin B_{k}\left(\gamma, p_{1}\right)}} v_{i_{1} i \gamma_{1}}<\Psi_{N}, \Phi_{h_{\tau}-\gamma^{\prime}-\gamma_{1}, i_{1}}> \\
&+O\left(\rho^{-p \alpha}\right) .
\end{aligned}
$$

In this manner, iterating $p_{1}$ times, we get

$$
\begin{aligned}
& \sum_{\substack{\gamma \prime \in \Gamma\left(\rho^{\alpha}\right) \\
h_{\tau}-\gamma^{\prime \notin B} B_{k}\left(\gamma, p_{1}\right)}} v_{i j \gamma^{\prime}}<\Psi_{N}, \Phi_{h_{\tau}-\gamma^{\prime}, i}>=\sum_{i_{1}, i_{2}, \ldots, i_{p_{1}}=1}^{m} \sum_{\substack{\gamma^{\prime}, \gamma_{1}, \gamma_{2}, \ldots, \gamma_{p_{1}} \in \Gamma\left(\rho^{\alpha}\right) \\
h_{\tau}-\gamma_{\prime}^{\prime} \notin B_{k}\left(\gamma, p_{1}\right)}}^{m} \\
& \frac{v_{i j \gamma} v_{i_{1} i \gamma_{1}} \ldots v_{i_{p_{1}} i_{p_{1}-1} \gamma_{p_{1}}}<\Psi_{N}, \Phi_{h_{\tau}-\gamma^{\prime}-\gamma_{1}-\cdots-\gamma_{p_{1}}, i_{p_{1}}}>}{\left(\Lambda_{N}-\left|h_{\tau}-\gamma^{\prime}\right|^{2}\right)\left(\Lambda_{N}-\left|h_{\tau}-\gamma^{\prime}-\gamma_{1}\right|^{2}\right) \ldots\left(\Lambda_{N}-\left|h_{\tau}-\gamma^{\prime}-\gamma_{1}-\cdots-\gamma_{p_{1}-1}\right|^{2}\right)} \\
& +O\left(\rho^{-p \alpha}\right) \text {. }
\end{aligned}
$$

Taking norm of both sides of the last equality, using $(19)$, the relation 8 and the fact that $p_{1} \alpha_{k+1} \geq p_{1} \alpha_{2}>p \alpha$, we obtain

$$
\text { | } \sum_{\substack{\gamma \prime \in \Gamma\left(\rho^{\alpha}\right) \\ h_{\tau}-\gamma^{\prime} \notin B_{k}\left(\gamma, p_{1}\right)}} v_{i j \gamma^{\prime}}<\Psi_{N}, \Phi_{h_{\tau}-\gamma \prime, i}>\mid=O\left(\rho^{-p \alpha}\right),
$$

which implies 20. Therefore, the equation 16 becomes

$$
\left(\Lambda_{N}-\left|h_{\tau}\right|^{2}\right)<\Psi_{N}, \Phi_{h_{\tau}, j}>=\sum_{i=1}^{m} \sum_{\substack{\gamma \prime \in \Gamma\left(\rho^{\alpha}\right) \\ h_{\tau}-\gamma^{\prime} \in B_{k}\left(\gamma, p_{1}\right)}} v_{i j \gamma^{\prime}}<\Psi_{N}, \Phi_{h_{\tau}-\gamma^{\prime}, i}>+O\left(\rho^{-p \alpha}\right) .
$$

Since $h_{\tau}-\gamma^{\prime} \in B_{k}\left(\gamma, p_{1}\right)$, using the notation $h_{\xi}=h_{\tau}-\gamma \prime$, the decomposition (22) can be written as

$$
\left(\Lambda_{N}-\left|h_{\tau}\right|^{2}\right)<\Psi_{N}, \Phi_{h_{\tau}, j}>=\sum_{i=1}^{m} \sum_{h_{\tau}-h_{\xi} \in \Gamma\left(\rho^{\alpha}\right)} v_{i j h_{\tau}-h_{\xi}}<\Psi_{N}, \Phi_{h_{\xi}, i}>+O\left(\rho^{-p \alpha}\right) .
$$


Isolating the terms where $h_{\tau}-h_{\xi}=0$ in 23 , we get

$$
\begin{aligned}
\left(\Lambda_{N}-\left|h_{\tau}\right|^{2}\right)<\Psi_{N}, \Phi_{h_{\tau}, j}> & =\sum_{i=1}^{m} v_{i j 0}<\Psi_{N}, \Phi_{h_{\tau}, i}> \\
& +\sum_{i=1}^{m} \sum_{\substack{h_{\tau}-h_{\xi} \in \Gamma\left(\rho^{\alpha}\right) \\
h_{\tau}-h_{\xi} \neq 0}} v_{i j h_{\tau}-h_{\xi}}<\Psi_{N}, \Phi_{h_{\xi}, i}> \\
& +O\left(\rho^{-p \alpha}\right) .
\end{aligned}
$$

Writing the equation (24) for all $j=1,2, \ldots, m$ and for any $\tau=1,2, \ldots, b_{k}$, , we get the system of equations

$$
\left[\left(\Lambda_{N}-\left|h_{\tau}\right|^{2}\right) I-V_{0}\right] A\left(N, h_{\tau}\right)=\sum_{\substack{\xi=1 \\ \xi \neq \tau}}^{b_{k}} V_{h_{\tau}-h_{\xi}} A\left(N, h_{\xi}\right)+O\left(\rho^{-p \alpha}\right),
$$

where $I$ is an $m \times m$ identity matrix, $V_{h_{\tau}-h_{\xi}}$ is given by 15],

$$
O\left(\rho^{-p \alpha}\right)=\left(O\left(\rho^{-p \alpha}\right), \ldots, O\left(\rho^{-p \alpha}\right)\right)
$$

is an $m \times 1$ vector and $A\left(N, h_{\xi}\right)$ is the $m \times 1$ vector

$$
A\left(N, h_{\xi}\right)=\left(<\Psi_{N}, \Phi_{h_{\xi}, 1}>,<\Psi_{N}, \Phi_{h_{\xi}, 2}>, \ldots,<\Psi_{N}, \Phi_{h_{\xi}, m}>\right)
$$

for any $\xi=1,2, \ldots, b_{k}$. Letting $\lambda_{N, \tau}=\Lambda_{N}-\left|h_{\tau}\right|^{2}$, we have

$$
\left[\begin{array}{cccc}
\lambda_{N, 1} I-V_{0} & -V_{h_{1}-h_{2}} & \cdots & -V_{h_{1}-h_{b_{k}}} \\
-V_{h_{2}-h_{1}} & \lambda_{N, 2} I-V_{0} & \cdots & -V_{h_{2}-h_{b_{k}}} \\
\vdots & & & \\
-V_{h_{b_{k}}-h_{1}} & -V_{h_{b_{k}}-h_{2}} & \cdots & \lambda_{N, b_{k}} I-V_{0}
\end{array}\right]\left[\begin{array}{c}
A\left(N, h_{1}\right) \\
A\left(N, h_{2}\right) \\
\vdots \\
A\left(N, h_{b_{k}}\right)
\end{array}\right]=\left[\begin{array}{c}
O\left(\rho^{-p \alpha}\right) \\
O\left(\rho^{-p \alpha}\right) \\
\vdots \\
O\left(\rho^{-p \alpha}\right),
\end{array}\right]
$$

We may write the system (27) as

$$
\left[\Lambda_{N} I-C\right] \mathcal{A}\left(N, h_{1}, h_{2}, \ldots, h_{b_{k}}\right)=\mathcal{O}\left(\rho^{-p \alpha}\right),
$$

where $I$ is an $m b_{k} \times m b_{k}$ identity matrix, $C$ is given by (14), $A\left(N, h_{1}, h_{2}, \ldots, h_{b_{k}}\right)$ is the $m b_{k} \times 1$ vector

$$
\mathcal{A}\left(N, h_{1}, h_{2}, \ldots, h_{b_{k}}\right)=\left(A\left(N, h_{1}\right), A\left(N, h_{2}\right), \ldots, A\left(N, h_{b_{k}}\right)\right)
$$

and the right side of the system $(28)$ is the $m b_{k} \times 1$ vector whose norm is

$$
\left|\mathcal{O}\left(\rho^{-p \alpha}\right)\right|=O\left(\sqrt{b_{k}} \rho^{-p \alpha}\right) .
$$

Theorem 1. Let $|\gamma|^{2}$ be a resonance eigenvalue of the operator $L(0)$, that is, $\gamma \in\left(\bigcap_{i=1}^{k} V_{\gamma_{i}}\left(\rho^{\alpha_{k}}\right)\right) \backslash E_{k+1}, k=1,2, \ldots, d-1$ where $|\gamma| \sim \rho$, and $\Lambda_{N}$ an eigenvalue 
of the operator $L(V)$ for which (18) holds and its corresponding eigenfunction $\Psi_{N}$ satisfies

$$
\left|<\Phi_{\gamma, j}, \Psi_{N}>\right|>c_{4} \rho^{-c \alpha} .
$$

Then there exists an eigenvalue $\eta_{s}(\gamma), 1 \leq s \leq m b_{k}$ of the matrix $C$ such that

$$
\Lambda_{N}=\eta_{s}(\gamma)+O\left(\rho^{-\left(p-c-\frac{d}{4} 3^{d}\right) \alpha}\right) .
$$

Proof. Since 18 is satisfied, 28 holds. Then multiplying both sides of the equation $(28)$ by $\left[\Lambda_{N} I-C\right]^{-1}$, then taking norm of both sides and by $(30)$, we get

$$
\left|\mathcal{A}\left(N, h_{1}, h_{2}, \ldots, h_{b_{k}}\right)\right| \leq\left\|\left[\Lambda_{N} I-C\right]^{-1}\right\| O\left(\sqrt{b_{k}} \rho^{-p \alpha}\right) .
$$

Using the fact that $\gamma$ is one of $h_{1}, h_{2}, \ldots, h_{\tau}$ (See definition of $\left.B_{k}\left(\gamma, p_{1}\right)\right)$ and hence by (31) and (32), we obtain

$$
c_{5} \rho^{-c \alpha}<\left|\mathcal{A}\left(N, h_{1}, h_{2}, \ldots, h_{b_{k}}\right)\right| \leq\left\|\left[\Lambda_{N} I-C\right]^{-1}\right\| \sqrt{b_{k}} c_{6} \rho^{-p \alpha} .
$$

Since $\left[\Lambda_{N} I-C\right]^{-1}$ is symmetric matrix with the eigenvalues $\frac{1}{\Lambda_{N}-\eta_{s}(\gamma)}, s=1, \ldots, m b_{k}$, we have

$$
\max _{s=1, \ldots, m b_{k}}\left|\Lambda_{N}-\eta_{s}(\gamma)\right|^{-1}=\left\|\left[\Lambda_{N} I-C\right]^{-1}\right\|>c_{7} c_{8}^{-1} b_{k}^{-\frac{1}{2}} \rho^{-c \alpha+p \alpha},
$$

where $b_{k}=O\left(\rho^{\frac{d}{2} 3^{d} \alpha}\right)$, thus

$$
\min _{s=1,2, \ldots, m b_{k}}\left|\Lambda_{N}-\eta_{s}\left(\gamma, \lambda_{i}\right)\right| \leq c_{9} \rho^{-\left(p-c-\frac{d}{4} 3^{d}\right) \alpha},
$$

and

$$
\Lambda_{N}=\eta_{s}\left(\gamma, \lambda_{i}\right)+O\left(\rho^{-\left(p-c-\frac{d}{4} 3^{d}\right) \alpha}\right)
$$

Theorem 2. Let $|\gamma|^{2}$ be a resonance eigenvalue of the operator $L(0)$, that is, $\gamma \in$ $\left(\bigcap_{i=1}^{k} V_{\gamma_{i}}\left(\rho^{\alpha_{k}}\right)\right) \backslash E_{k+1}, k=1,2, \ldots, d-1$ where $|\gamma| \sim \rho, \eta_{s}(\gamma)$ an eigenvalue of the matrix $C$ such that $\left.\left|\eta_{s}(\gamma)-\right| \gamma\right|^{2} \mid<\frac{3}{8} \rho^{\alpha_{1}}$. Then there is an eigenvalue $\Lambda_{N}$ of the operator $L(V)$ satisfying

$$
\Lambda_{N}=\eta_{s}(\gamma)+O\left(\rho^{-p \alpha+\frac{d}{4} 3^{d} \alpha+\frac{d-1}{2}}\right) .
$$

Proof. By the general perturbation theory, there is an eigenvalue $\Lambda_{N}$ of the operator $L(V)$ such that $\left.\left|\Lambda_{N}-\right| \gamma\right|^{2} \mid<\frac{1}{2} \rho^{2 \alpha_{1}}$ holds. Thus one can use the system (28) and we prove the theorem for this eigenvalue $\Lambda_{N}$ :

Let $\eta_{s}, s=1,2, \ldots, m b_{k}$ be an eigenvalue of the matrix $C$ and $\theta_{s}=\left(\theta_{s}^{1}, \theta_{s}^{2}, \ldots, \theta_{s}^{b_{k}}\right)_{m b_{k} \times 1}$ the corresponding normalized eigenvector, where $\theta_{s}^{\tau}=\left(\theta_{s}^{\tau 1}, \theta_{s}^{\tau 2}, \ldots, \theta_{s}^{\tau m}\right)_{m \times 1}, \tau=1,2, \ldots, b_{k}$. Multiplying the equation 28) by $\theta_{s}$, since $C$ is symmetric (see (14) and (15)), we get

$$
\left|\Lambda_{N}-\eta_{s}\right|\left|\mathcal{A}\left(N, h_{1}, h_{2}, \ldots, h_{b_{k}}\right) \cdot \theta_{s}\right|=\left|\mathcal{O}\left(\rho^{-p \alpha}\right) \cdot \theta_{s}\right| .
$$


By using $b_{k}=O\left(\rho^{\frac{d}{2} 3^{d} \alpha}\right)$, (30) and the Cauchy Schwartz Inequality for the right hand side of (34), we have

$$
\left|\Lambda_{N}-\eta_{s}\right|\left|\mathcal{A}\left(N, h_{1}, h_{2}, \ldots, h_{b_{k}}\right) \cdot \theta_{s}\right|=O\left(\rho^{-p \alpha+\frac{d}{4} 3^{d} \alpha}\right) .
$$

So we need to prove that

$$
\left|\mathcal{A}\left(N, h_{1}, h_{2}, \ldots, h_{b_{k}}\right) \cdot \theta_{s}\right|>c_{10} \rho^{-\frac{d-1}{2}},
$$

from which the theorem follows.

For this purpose,we first consider the decomposition of the matrix $C$ as $C=$ $A+B$, where

$$
A=\left[\begin{array}{ccc}
\left|h_{1}\right|^{2} I & & 0 \\
& \ddots & \\
0 & & \left|h_{b_{k}}\right|^{2} I
\end{array}\right], \quad B=\left[\begin{array}{cccc}
V_{0} & V_{h_{1}-h_{2}} & \cdots & V_{h_{1}-h_{b_{k}}} \\
V_{h_{2}-h_{1}} & V_{0} & \cdots & V_{h_{2}-h_{b_{k}}} \\
\vdots & & \ddots & \vdots \\
V_{h_{b_{k}}-h_{1}} & V_{h_{b_{k}}-h_{2}} & \cdots & V_{0}
\end{array}\right] .
$$

The eigenvalues and the corresponding eigenspaces of the matrix $A$ are $\left|h_{\tau}\right|^{2}$ and $E_{\tau}=\operatorname{span}\left\{e_{j}:(\tau-1) m+1 \leq j \leq \tau m\right\}$, respectively, where

$$
\left\{e_{j}=(0, \ldots, 0,1,0, \ldots, 0)\right\}_{j=1}^{m b_{k}}
$$

is the standard basis of $R^{m b_{k}}$. Now, we use the following notation

$$
\theta_{s}\left(h_{\tau, j}\right) \equiv \theta_{s} \cdot e_{j}=\theta_{s}^{\tau j}, \quad \text { if } \quad(\tau-1) m+1 \leq j \leq \tau m
$$

for $\tau=1,2, \cdots, b_{k}$.

Multiplying $(A+B) \theta_{s}=\eta_{s} \theta_{s}$ by $e_{j}$, since $A$ and $B$ are symmetric, we get

$$
\left(\eta_{s}-\left|h_{\tau}\right|^{2}\right) \theta_{s}\left(h_{\tau, j}\right)=\theta_{s} \cdot B e_{j}
$$

and $(\tau-1) m+1 \leq j \leq \tau m$, and $\tau=1,2, \cdots, b_{k}$.

On the other hand, if we consider the sum of the elements in the i-th row of the matrix $B$, by 8

$$
\sum_{\substack{\tau=1 \\ \tau \neq i}}^{b_{k}} \sum_{j=1}^{m} v_{i j h_{i}-h_{\tau}}<\sum_{j=1}^{m} M_{i j}
$$

for all $i=1,2, \ldots, m$. Since $B$ is a symmetric matrix and by 40 , the sum of elements in each row of $B$ is less then $M=\max _{i=1,2, \ldots, m}\left\{\sum_{j=1}^{m} M_{i j}\right\}$, the eigenvalues of $B$ are also less then $M$ from which we have $\|B\| \leq M$.

Thus, by 26), 36), 38, we have

$$
\left|\mathcal{A}\left(N, h_{1}, \ldots, h_{b_{k}}\right) \cdot \theta_{s}\right|=\left|\left\langle\psi_{N}, \sum_{\tau=1}^{b_{k}} \sum_{j=1}^{m} \theta_{s}\left(h_{\tau, j}\right) \phi_{h_{\tau, j}}\right\rangle\right|
$$


which, together with Parseval's relation, imply

$$
\begin{aligned}
1= & \left\|\sum_{\tau=1}^{b_{k}} \sum_{i=1}^{m} \theta_{s}\left(h_{\tau, i}\right) \Phi_{h_{\tau}, i}\right\|^{2} \\
= & \sum_{N:\left.\left|\Lambda_{N}-\right| \gamma\right|^{2} \mid \geq \frac{1}{2} \rho^{2 \alpha_{1}}}\left|\sum_{\tau=1}^{b_{k}} \sum_{i=1}^{m} \theta_{s}\left(h_{\tau, i}\right)<\Psi_{N}, \Phi_{h_{\tau}, i}>\right|^{2} \\
& \quad+\sum_{N:\left.\left|\Lambda_{N}-\right| \gamma\right|^{2} \mid<\frac{1}{2} \rho^{2 \alpha_{1}}}\left|\sum_{\tau=1}^{b_{k}} \sum_{i=1}^{m} \theta_{s}\left(h_{\tau, i}\right)<\Psi_{N}, \Phi_{h_{\tau}, i}>\right|^{2} .
\end{aligned}
$$

Now we estimate the first summation in the expression 42 :

$$
\begin{aligned}
& \sum_{N:\left.\left|\Lambda_{N}-\right| \gamma\right|^{2} \mid \geq \frac{1}{2} \rho^{2 \alpha_{1}}}\left|\sum_{\tau=1}^{b_{k}} \sum_{i=1}^{m} \theta_{s}\left(h_{\tau, i}\right)<\Psi_{N}, \Phi_{h_{\tau}, i}>\right|^{2} \\
& =\sum_{N:\left.\left|\Lambda_{N}-\right| \gamma\right|^{2} \mid \geq \frac{1}{2} \rho^{2 \alpha_{1}}} \mid \sum_{\tau:\left.\left|\eta_{s}-\right| h_{\tau}\right|^{2} \mid<\frac{1}{8} \rho^{\alpha_{1}}} \sum_{i=1}^{m} \theta_{s}\left(h_{\tau, i}\right)<\Psi_{N}, \Phi_{h_{\tau}, i}> \\
& +\sum_{\tau:\left.\left|\eta_{s}-\right| h_{\tau}\right|^{2} \mid \geq \frac{1}{8} \rho^{\alpha_{1}}} \sum_{i=1}^{m} \theta_{s}\left(h_{\tau, i}\right)<\Psi_{N}, \Phi_{h_{\tau}, i}>\left.\right|^{2} \\
< & 2 \sum_{N:\left.\left|\Lambda_{N}-\right| \gamma\right|^{2} \mid \geq \frac{1}{2} \rho^{2 \alpha_{1}}}\left|\sum_{\tau:\left.\left|\eta_{s}-\right| h_{\tau}\right|^{2} \mid<\frac{1}{8} \rho^{\alpha_{1}}} \sum_{i=1}^{m} \theta_{s}\left(h_{\tau, i}\right)<\Psi_{N}, \Phi_{h_{\tau}, i}>\right|^{2} \\
& +2 \sum_{N:\left.\left|\Lambda_{N}-\right| \gamma\right|^{2} \mid \geq \frac{1}{2} \rho^{2 \alpha_{1}}}\left|\sum_{\tau:\left.\left|\eta_{s}-\right| h_{\tau}\right|^{2} \mid \geq \frac{1}{8} \rho^{\alpha_{1}}} \sum_{i=1}^{m} \theta_{s}\left(h_{\tau, i}\right)<\Psi_{N}, \Phi_{h_{\tau}, i}>\right|^{2} .
\end{aligned}
$$

Using Bessel's inequality, Parseval's relation, orthogonality of the functions $\Phi_{h_{\tau}, i}(x)$, $\tau=1,2, \ldots, b_{k}, i=1,2, \ldots, m$, the binding formula (39) and $\|B\| \leq M$, we have

$$
\begin{aligned}
\sum_{N:\left.\left|\Lambda_{N}-\right| \gamma\right|^{2} \mid \geq \frac{1}{2} \rho^{2 \alpha_{1}}}\left|\sum_{\tau:\left.\left|\eta_{s}-\right| h_{\tau}\right|^{2} \mid \geq \frac{1}{8} \rho^{\alpha_{1}}} \sum_{i=1}^{m} \theta_{s}\left(h_{\tau, i}\right)<\Psi_{N}, \Phi_{h_{\tau}, i}>\right|^{2} \\
\leqslant\left\|\sum_{\tau:\left.\left|\eta_{s}-\right| h_{\tau}\right|^{2} \mid \geq \frac{1}{8} \rho^{\alpha_{1}}} \sum_{i=1}^{m} \theta_{s}\left(h_{\tau, i}\right) \Phi_{h_{\tau}, i}\right\|^{2} \\
=\sum_{\tau:\left.\left|\eta_{s}-\right| h_{\tau}\right|^{2} \mid \geq \frac{1}{8} \rho^{\alpha_{1}}} \sum_{i=1}^{m}\left|\theta_{s}\left(h_{\tau, i}\right)\right|^{2}\left\|\Phi_{h_{\tau}, i}\right\|^{2} \\
=\sum_{\tau:\left.\left|\eta_{s}-\right| h_{\tau}\right|^{2} \mid \geq \frac{1}{8} \rho^{\alpha_{1}}} \sum_{i=1}^{m} \frac{\left|\theta_{s} \cdot B e_{i}\right|^{2}}{\left.\left.\left|\eta_{s}-\right| h_{\tau}\right|^{2}\right|^{2}}=O\left(\rho^{-2 \alpha_{1}}\right) .
\end{aligned}
$$


The assumption $\left.\left|\eta_{s}-\right| \gamma\right|^{2} \mid<\frac{3}{8} \rho^{\alpha_{1}}$ of the theorem and $\left.\left|\eta_{s}-\right| h_{\tau}\right|^{2} \mid<\frac{1}{8} \rho^{\alpha_{1}}$ imply that $\left.|| \gamma\right|^{2}-\left|h_{\tau}\right|^{2} \mid<\frac{1}{2} \rho^{\alpha_{1}}$. So by the well-known formula

$$
\frac{1}{\Lambda_{N}-\left|h_{\tau}\right|^{2}}=\frac{1}{\Lambda_{N}-|\gamma|^{2}}\left\{\sum_{n=0}^{k}\left(\frac{\left|h_{\tau}\right|^{2}-|\gamma|^{2}}{\Lambda_{N}-|\gamma|^{2}}\right)^{n}+O\left(\rho^{-(k+1) \alpha_{1}}\right)\right\}
$$

for $\left.\left|\Lambda_{N}-\right| \gamma\right|^{2} \mid \geq \frac{1}{2} \rho^{2 \alpha_{1}}$, and $\left.|| \gamma\right|^{2}-\left|h_{\tau}\right|^{2} \mid<\frac{1}{2} \rho^{2 \alpha_{1}}$, using (39), we have

$$
\begin{aligned}
& \sum_{N:\left.\left|\Lambda_{N}-\right| \gamma\right|^{2} \mid \geq \frac{1}{2} \rho^{2 \alpha_{1}}}\left|\sum_{\tau:\left.\left|\eta_{s}-\right| h_{\tau}\right|^{2} \mid<\frac{1}{8} \rho^{\alpha_{1}}} \sum_{i=1}^{m} \theta_{s}\left(h_{\tau, i}\right)<\Psi_{N}, \Phi_{h_{\tau}, i}>\right|^{2} \\
& =\sum_{N:\left.\left|\Lambda_{N}-\right| \gamma\right|^{2} \mid \geq \frac{1}{2} \rho^{2 \alpha_{1}}}\left|\sum_{\tau:\left.\left|\eta_{s}-\right| h_{\tau}\right|^{2} \mid<\frac{1}{8} \rho^{\alpha_{1}}} \sum_{i=1}^{m} \theta_{s}\left(h_{\tau, i}\right) \frac{<\Psi_{N}, V \Phi_{h_{\tau}, i}>}{\Lambda_{N}-\left|h_{\tau}\right|^{2}}\right|^{2} \\
& \leq \sum_{N:\left.\left|\Lambda_{N}-\right| \gamma\right|^{2} \mid \geq \frac{1}{2} \rho^{2 \alpha_{1}}}(k+1)\left|\sum_{\tau:\left.\left|\eta_{s}-\right| h_{\tau}\right|^{2} \mid<\frac{1}{8} \rho^{\alpha_{1}}} \sum_{i=1}^{m} \frac{\theta_{s}\left(h_{\tau, i}\right)<\Psi_{N}, V \Phi_{h_{\tau}, i}>}{\Lambda_{N}-|\gamma|^{2}}\right|^{2} \\
& +\sum_{N:\left.\left|\Lambda_{N}-\right| \gamma\right|^{2} \mid \geq \frac{1}{2} \rho^{2 \alpha_{1}}}(k+1)\left|\sum_{\tau:\left.\left|\eta_{s}-\right| h_{\tau}\right|^{2} \mid<\frac{1}{8} \rho^{\alpha_{1}}} \sum_{i=1}^{m} \frac{\theta_{s}\left(h_{\tau, i}\right)<\Psi_{N}, V \Phi_{h_{\tau}, i}>}{\Lambda_{N}-|\gamma|^{2}} \frac{\left|h_{\tau}\right|^{2}-|\gamma|^{2}}{\Lambda_{N}-|\gamma|^{2}}\right|^{2} \\
& +\sum_{N:\left.\left|\Lambda_{N}-\right| \gamma\right|^{2} \mid \geq \frac{1}{2} \rho^{2 \alpha_{1}}}(k+1)\left|\sum_{\tau:\left.\left|\eta_{s}-\right| h_{\tau}\right|^{2} \mid<\frac{1}{8} \rho^{\alpha_{1}}} \sum_{i=1}^{m} \frac{\theta_{s}\left(h_{\tau, i}\right)<\Psi_{N}, V \Phi_{h_{\tau}, i}>}{\Lambda_{N}-|\gamma|^{2}}\left[\frac{\left.h_{\tau}\right|^{2}-|\gamma|^{2}}{\Lambda_{N}-|\gamma|^{2}}\right]^{k}\right|^{2} \\
& +\sum_{N:\left.\left|\Lambda_{N}-\right| \gamma\right|^{2} \mid \geq \frac{1}{2} \rho^{2 \alpha_{1}}}(k+1)\left|\sum_{\tau:\left.\left|\eta_{s}-\right| h_{\tau}\right|^{2} \mid<\frac{1}{8} \rho^{\alpha_{1}}} \sum_{i=1}^{m} \theta_{s}\left(h_{\tau, i}\right)<\Psi_{N}, V \Phi_{h_{\tau}, i}>O\left(\rho^{-(k+1) \alpha_{1}}\right)\right|^{2} .
\end{aligned}
$$

To calculate the order of each term in (44), we use Bessel's inequality and the orthogonality of $\Phi_{h_{\tau}, i}$. So we have

$$
\begin{aligned}
& 2 \sum_{N:\left.\left|\Lambda_{N}-\right| \gamma\right|^{2} \mid \geq \frac{1}{2} \rho^{2 \alpha_{1}}}(k+1) \\
& \times \sum_{\tau:\left.\left|\eta_{s}-\right| h_{\tau}\right|^{2} \mid<\frac{1}{8} \rho^{\alpha_{1}}} \sum_{i=1}^{m} \theta_{s}\left(h_{\tau, i}\right)<\Psi_{N}, V \Phi_{h_{\tau}, i}>\left.\frac{\left(\left|h_{\tau}\right|^{2}-|\gamma|^{2}\right)^{r}}{\left(\Lambda_{N}-|\gamma|^{2}\right)^{r+1}}\right|^{2} \\
= & 2 \sum_{N:\left.\left|\Lambda_{N}-\right| \gamma\right|^{2} \mid \geq \frac{1}{2} \rho^{2 \alpha_{1}}} \frac{(k+1)}{\left.\left.\left|\Lambda_{N}-\right| \gamma\right|^{2}\right|^{2(r+1)}}
\end{aligned}
$$




$$
\begin{aligned}
& \times\left|\sum_{\tau:\left.\left|\eta_{s}-\right| h_{\tau}\right|^{2} \mid<\frac{1}{8} \rho^{\alpha_{1}}} \sum_{i=1}^{m} \theta_{s}\left(h_{\tau, i}\right)<\Psi_{N}, V \Phi_{h_{\tau}, i}>\left(\left|h_{\tau}\right|^{2}-|\gamma|^{2}\right)^{r}\right|^{2} \\
\leq & c_{11}\left(\rho^{2 \alpha_{1}}\right)^{-2(r+1)}(k+1) \\
& \times \sum_{N:\left.\left|\Lambda_{N}-\right| \gamma\right|^{2} \mid \geq \frac{1}{2} \rho^{2 \alpha_{1}}}\left|<\Psi_{N}, \sum_{\tau:\left.\left|\eta_{s}-\right| h_{\tau}\right|^{2} \mid<\frac{1}{8} \rho^{\alpha_{1}}} \sum_{i=1}^{m} \theta_{s}\left(h_{\tau, i}\right)\left(\left|h_{\tau}\right|^{2}-|\gamma|^{2}\right)^{r} V \Phi_{h_{\tau}, i}>\right|^{2} \\
\leq & c_{12}\left(\rho^{2 \alpha_{1}}\right)^{-2(r+1)}(k+1)\left|\sum_{\tau:\left.\left|\eta_{s}-\right| h_{\tau}\right|^{2} \mid<\frac{1}{8} \rho^{\alpha_{1}}} \sum_{i=1}^{m} \theta_{s}\left(h_{\tau, i}\right)\left(\left|h_{\tau}\right|^{2}-|\gamma|^{2}\right)^{r} V \Phi_{h_{\tau}, i} \|\right|^{2} \\
\leq & c_{13}\left(\rho^{2 \alpha_{1}}\right)^{-2(r+1)}(k+1)\left(\sum_{\tau:\left.\left|\eta_{s}-\right| h_{\tau}\right|^{2} \mid<\frac{1}{8} \rho^{\alpha_{1}}} \sum_{i=1}^{m}\left\|\theta_{s}\left(h_{\tau, i}\right)\left(\left|h_{\tau}\right|^{2}-|\gamma|^{2}\right)^{r} V \Phi_{h_{\tau}, i}\right\|\right)^{2} \\
= & c_{14}\left(\rho^{2 \alpha_{1}}\right)^{-2(r+1)}(k+1)\left(\left.\sum_{\tau:\left.\left|\eta_{s}-\right| h_{\tau}\right|^{2} \mid<\frac{1}{8} \rho^{\alpha_{1}}} \sum_{i=1}^{m}\left|\theta_{s}\left(h_{\tau, i}\right)\right||| h_{\tau}\right|^{2}-\left.|\gamma|^{2}\right|^{r}\left\|V \Phi_{h_{\tau}, i}\right\|\right)^{2} \\
\leq & c_{15}\left(\rho^{2 \alpha_{1}}\right)^{-2(r+1)}\left(\frac{1}{2} \rho^{\alpha_{1}}\right)^{2 r}(k+1)\left(\sum_{\tau:\left.\left|\eta_{s}-\right| h_{\tau}\right|^{2} \mid<\frac{1}{8} \rho^{\alpha_{1}}} \sum_{i=1}^{m}\left\|V \Phi_{h_{\tau}, i}\right\|\right)^{2}=O\left(\rho^{-2(r+1) \alpha_{1}}\right),
\end{aligned}
$$

for $r=0,1,2, \ldots, k$. Now let $K$ be the number of $h_{\tau}$ satisfying $\left.\left|\eta_{s}-\right| h_{\tau}\right|^{2} \mid<$ $\frac{1}{8} \rho^{\alpha_{1}}$, then the order of the last summation in 46 is:

$$
\begin{aligned}
& \sum_{N:\left.\left|\Lambda_{N}-\right| \gamma\right|^{2} \mid \geq \frac{1}{2} \rho^{2 \alpha_{1}}}(k+1) \\
& \quad \times\left|\sum_{\tau:\left.\left|\eta_{s}-\right| h_{\tau}\right|^{2} \mid<\frac{1}{8} \rho^{\alpha_{1}}} \sum_{i=1}^{m} \theta_{s}\left(h_{\tau, i}\right)<\Psi_{N}, V \Phi_{h_{\tau}, i}>O\left(\rho^{-(k+1) \alpha_{1}}\right)\right|^{2} \\
& \quad K \sum_{N:\left.\left|\Lambda_{N}-\right| \gamma\right|^{2} \mid \geq \frac{1}{2} \rho^{2 \alpha_{1}}}(k+1) \\
& \quad \times \sum_{\tau:\left.\left|\eta_{s}-\right| h_{\tau}\right|^{2} \mid<\frac{1}{8} \rho^{\alpha_{1}}}\left|O\left(\rho^{-(k+1) \alpha_{1}}\right)\right|^{2} \cdot\left|\theta_{s}\left(h_{\tau, i}\right)\right|^{2} \cdot\left|<\Psi_{N}, V \Phi_{h_{\tau}, i}>\right|^{2} \\
& \leq c_{16} \cdot K \cdot \rho^{-2(k+1) \alpha_{1}} \cdot \sum_{\tau:\left.\left|\eta_{s}-\right| h_{\tau}\right|^{2} \mid<\frac{1}{8} \rho^{\alpha_{1}}}\left\|V(x) \Phi_{h_{\tau}, i}\right\|^{2} \\
& \leq c_{17} \cdot K^{2} \cdot M^{2} \cdot \rho^{-2(k+1) \alpha_{1}}=K^{2} \cdot 0\left(\rho^{-2(k+1) \alpha_{1}}\right)=O\left(\rho^{-2 \alpha_{1}}\right),
\end{aligned}
$$


since $K=O\left(\rho^{\frac{d}{2} \alpha_{d}}\right)$ and we can always choose $k$ in $O\left(\rho^{-2(k+1) \alpha_{1}}\right)$ such that

$$
K^{2} \cdot O\left(\rho^{-2(k+1) \alpha_{1}}\right)=O\left(\rho^{-2 \alpha_{1}}\right),
$$

which together with the estimations 44, , 45 and (46) imply

$$
O\left(\rho^{-2 \alpha_{1}}\right)=\sum_{N:\left.\left|\Lambda_{N}-\right| \gamma\right|^{2} \mid \geq \frac{1}{2} \rho^{2 \alpha_{1}}}\left|\sum_{\tau=1}^{b_{k}} \sum_{i=1}^{m} \theta_{s}\left(h_{\tau, i}\right)<\Psi_{N}, \Phi_{h_{\tau}, i}>\right|^{2} .
$$

Therefore, from the decomposition 42 we have

$$
1-O\left(\rho^{-2 \alpha_{1}}\right)=\sum_{N:\left.\left|\Lambda_{N}-\right| \gamma\right|^{2} \mid<\frac{1}{2} \rho^{2 \alpha_{1}}}\left|\sum_{\tau=1}^{b_{k}} \sum_{i=1}^{m} \theta_{s}\left(h_{\tau, i}\right)<\Psi_{N}, \Phi_{h_{\tau}, i}>\right|^{2} .
$$

Since the number of indexes $N$ satisfying $\left.\left|\Lambda_{N}-\right| \gamma\right|^{2} \mid<\frac{1}{2} \rho^{2 \alpha_{1}}$ is less then $\rho^{d-1}$, we have

$$
1-O\left(\rho^{-2 \alpha_{1}}\right) \leq \rho^{d-1} \max _{N:\left.\left|\Lambda_{N}-\right| \gamma\right|^{2} \mid<\frac{1}{2} \rho^{2 \alpha_{1}}}\left\{\left|\sum_{\tau=1}^{b_{k}} \sum_{i=1}^{m} \theta_{s}\left(h_{\tau, i}\right)<\Psi_{N}, \Phi_{h_{\tau}, i}>\right|^{2}\right\}
$$

which implies together with the relation (41) that

$$
\left|A\left(N, h_{1}, h_{2}, \ldots, h_{b_{k}}\right) \cdot \theta_{s}\right|^{2} \geq \frac{1-O\left(\rho^{-2 \alpha_{1}}\right)}{\rho^{d-1}} .
$$

It follows from the equation 35 and the estimation 48 that

$$
\Lambda_{N}=\eta_{s}+\frac{O\left(\rho^{-p \alpha+\frac{d}{4} 3^{d} \alpha}\right)}{O\left(\rho^{-\frac{d-1}{2}}\right)}
$$

that is, 36 holds.

\section{Asymptotic Formulas for the Eigenvalues in A Single Resonance Domain}

Now, we investigate in detail the eigenvalues of $L(V)$ in a single resonance domain. In order the inequalities

$$
0<\alpha<\frac{1}{d+20}, \quad 2 \alpha_{2}-\alpha_{1}+(d+3) \alpha<1
$$

and

$$
\alpha_{2}>2 \alpha_{1}
$$

to be satisfied, we can choose $\alpha, \alpha_{1}$ and $\alpha_{2}$ as follows

$$
\alpha=\frac{1}{d+p}, \quad \alpha_{1}=\frac{p_{2}}{d+p}, \quad \alpha_{2}=\frac{2 p_{2}+1}{d+p},
$$


where $p_{2}=\left[\frac{p-5}{3}\right]-1$. Let $\gamma \in V_{\delta}\left(\rho^{\alpha_{1}}\right) \backslash E_{2}, \delta \in \frac{\Gamma}{2} \backslash\left\{e_{i}\right\}$, where $\delta$ is minimal in its direction. Consider the following sets :

$$
\begin{aligned}
& B_{1}(\delta)=\left\{b: b=n \delta, n \in Z,|b|<\frac{1}{2} \rho^{\frac{1}{2} \alpha_{2}}\right\}, \\
& B_{1}(\gamma)=\gamma+B_{1}(\delta)=\left\{\gamma+b: b \in B_{1}(\delta)\right\}, \\
& B_{1}\left(\gamma, p_{1}\right)=B_{1}(\gamma)+\Gamma\left(p_{1} \rho^{\alpha}\right) .
\end{aligned}
$$

As before, denote by $h_{\tau}, \tau=1,2, \ldots, b_{1}$ the vectors of $B_{1}\left(\gamma, p_{1}\right)$, where $b_{1}$ is the number of vectors in $B_{1}\left(\gamma, p_{1}\right)$. Then the matrix $C(\gamma, \delta)=\left(c_{i j}\right), i, j=1,2, \ldots, m b_{1}$ is defined by

$$
C(\gamma, \delta)=\left[\begin{array}{cccc}
\left|h_{1}\right|^{2} I-V_{0} & V_{h_{1}-h_{2}} & \cdots & V_{h_{1}-h_{b_{1}}} \\
V_{h_{2}-h_{1}} & \left|h_{2}\right|^{2} I-V_{0} & \cdots & V_{h_{2}-h_{b_{1}}} \\
\vdots & & & \\
V_{h_{b_{1}}-h_{1}} & V_{h_{b_{1}}-h_{2}} & \cdots & \left|h_{b_{1}}\right|^{2} I-V_{0}
\end{array}\right],
$$

where $V_{h_{\tau}-h_{\xi}}, \tau, \xi=1,2, \ldots, b_{1}$ are the $m \times m$ matrices defined by 15 ).

Also we define the matrix $D(\gamma, \delta)=\left(c_{i j}\right)$ for $i, j=1,2, \ldots, m a_{1}$, where $h_{1}, h_{2}, \ldots, h_{a_{1}}$ are the vectors of $B_{1}\left(\gamma, p_{1}\right) \bigcap\{\gamma+n \delta: n \in Z\}$, and $a_{1}$ is the number of vectors in $B_{1}\left(\gamma, p_{1}\right) \bigcap\{\gamma+n \delta: n \in Z\}$. Clearly $a_{1}=O\left(\rho^{\frac{1}{2} \alpha_{2}}\right)$.

Lemma 3. a) If $\eta_{j_{s}}$ is an eigenvalue of the matrix $C(\gamma, \delta)$ such that $\left.\left|\eta_{j_{s}}-\right| h_{s}\right|^{2} \mid<$ $M$ for $s=1,2, \ldots, a_{1}, 1+(s-1) m \leq j_{s} \leq m s$, then

$$
\left.\left|\eta_{j_{s}}-\right| h_{\tau}\right|^{2} \mid>\frac{1}{4} \rho^{\alpha_{2}}, \forall \tau=a_{1}+1, a_{1}+2, \ldots, b_{1} .
$$

b) If $\eta_{j_{s}}$ is an eigenvalue of the matrix $C(\gamma, \delta)$ such that $\left.\left|\eta_{j_{s}}-\right| h_{s}\right|^{2} \mid<M$ for $s=a_{1}+1, a_{1}+2, \ldots, b_{1}$ and $1+(s-1) m \leq j_{s} \leq m s$, then

$$
\left.\left|\eta_{j_{s}}-\right| h_{\tau}\right|^{2} \mid>\frac{1}{4} \rho^{\alpha_{2}}, \forall \tau=1,2, \ldots, a_{1} .
$$

Proof. First we prove

$$
\left.|| h_{\tau}\right|^{2}-\left|h_{s}\right|^{2} \mid \geq \frac{1}{3} \rho^{\alpha_{2}}, \quad \forall s \leq a_{1}, \quad \forall \tau>a_{1} .
$$

By definition, if $s \leq a_{1}$ then $h_{s}=\gamma+n \delta$, where $|n \delta|<\frac{1}{2} \rho^{\frac{1}{2} \alpha_{2}}+p_{1} \rho^{\alpha}$. If $\tau>a_{1}$ then $h_{\tau}=\gamma+s^{\prime} \delta+a$, where $\left|s^{\prime} \delta\right|<\frac{1}{2} \rho^{\frac{1}{2} \alpha_{2}}, a \in \Gamma\left(p_{1} \rho^{\alpha}\right) \backslash \delta R$. Therefore

$$
\left|h_{\tau}\right|^{2}-\left|h_{s}\right|^{2}=2 \gamma \cdot a+2 s^{\prime} \delta \cdot a+2 s^{\prime} \gamma \cdot \delta+\left|s^{\prime} \delta\right|^{2}+|a|^{2}-2 n \gamma \cdot \delta-|n \delta|^{2} .
$$

Since $\gamma \notin V_{a}\left(\rho^{\alpha_{2}}\right),|a|<p_{1} \rho^{\alpha}$, we have

$$
|2 \gamma \cdot a|>\rho^{\alpha_{2}}-c_{0} \rho^{2 \alpha} .
$$

The relation $\gamma \in V_{\delta}\left(\rho^{\alpha_{1}}\right)$ and the inequalities for $s^{\prime}$ and $n$ imply that

$$
2 s^{\prime} \gamma \cdot \delta+2 s^{\prime} \gamma \cdot a+|a|^{2}-2 n \gamma \cdot \delta=O\left(\rho^{\frac{1}{2} \alpha_{2}+\alpha_{1}}\right),
$$




$$
\left.|| s^{\prime} \delta\right|^{2}-|n \delta|^{2} \mid<\frac{1}{4} \rho^{\alpha_{2}}+c_{0} \rho^{\frac{1}{2} \alpha_{2}+\alpha} .
$$

Thus 52 follows from these relations, since $\frac{1}{2} \alpha_{2}+\alpha_{1}<\alpha_{2}$ and $\frac{1}{2} \alpha_{2}+\alpha<\alpha_{2}$.

The eigenvalues of $D(\gamma, \delta)$ and $C(\gamma, \delta)$ lay in $M$-neighborhood of the numbers $\left|h_{k}\right|^{2}$ for $k=1,2, \ldots, a_{1}$ and for $k=1,2, \ldots, b_{1}$, respectively. The inequality (52) shows that one can enumerate the eigenvalues $\eta_{j}\left(j=1,2, \ldots, m b_{1}\right)$ of $C$ in the following way:

$$
\eta_{j} \equiv \eta_{j_{s}}, \quad j_{s} \leq m a_{1}, \quad 1+(s-1) m \leq j_{s} \leq s m
$$

when for $s \leq a_{1}, \eta_{j}$ lay in M-neighborhood of $\left|h_{s}\right|^{2}$ and

$$
\eta_{j} \equiv \eta_{j_{\tau}}, \quad j_{\tau} \geq m a_{1}, \quad 1+(\tau-1) m \leq j_{\tau} \leq \tau m
$$

when for $\tau>a_{1}, \eta_{j}$ lay in M-neighborhood $\left|h_{\tau}\right|^{2}$. Then by $(52$, we get

$$
\left.\left|\eta_{j_{s}}-\right| h_{\tau}\right|^{2} \mid>\frac{1}{4} \rho^{\alpha_{2}}
$$

for $s \leq a_{1}, \tau>a_{1}$ and $s>a_{1}, \tau \leq a_{1}$.

Now, using the notation $h_{s}=\gamma-\left(\frac{s}{2}\right) \delta$ if $s$ is even, $h_{s}=\gamma+\left(\frac{s-1}{2}\right) \delta$ if $s$ is odd, for $s=1,2, \ldots, a_{1}$, (without loss of generality assume that $a_{1}$ is even) and using the orthogonal decomposition of $\gamma \in \frac{\Gamma}{2}, \gamma=\beta+(l+v(\beta)) \delta$, where $\beta \in H_{\delta} \equiv\left\{x \in R^{d}\right.$ : $x \cdot \delta=0\}, l \in Z, v \in[0,1)$ we can write the matrix $D(\gamma, \delta)$ as

$$
D(\gamma, \delta)=|\beta|^{2} I+E(\gamma, \delta),
$$

where $I$ is a maximal identity matrix and $E(\gamma, \delta)$ is

$$
E(\gamma, \delta)=\left[\begin{array}{ccccc}
\left((l+v)^{2}|\delta|^{2}\right) I+V_{0} & V_{\delta} & V_{-\delta} & \cdots & V_{\frac{a_{1}}{2} \delta} \\
V_{-\delta} & \left((l-1+v)^{2}|\delta|^{2}\right) I+V_{0} & V_{-2 \delta} & \cdots & V^{\left(\frac{a_{1}}{2}-1\right) \delta} \\
V_{\delta} & V_{2 \delta} & \left.(l+1+v)^{2}|\delta|^{2}\right) I+V_{0} & \cdots & V_{\left(\frac{a_{1}}{2}+1\right) \delta} \\
\vdots & \vdots & \vdots & \vdots & \vdots \\
V_{-\frac{a_{1}}{2} \delta} & \vdots & \vdots & \cdots & \left.\left(l-\frac{a_{1}}{2}+v\right)^{2}|\delta|^{2}\right) I+V_{0}
\end{array}\right]
$$

Denote $n_{k}=-\frac{k}{2}$ if $k$ is even, $n_{k}=\frac{k-1}{2}$ if $k$ is odd. The system $\left\{e^{i\left(n_{k}+v\right) t}: k=1,2, \ldots\right\}$ is a basis in $L_{2}^{m}[0,2 \pi]$. Let $T(\gamma, \delta) \equiv T(P(t), \beta)$ be the operator in $\ell_{2}$ corresponding to the Sturm-Liouville operator $T$, generated by

$$
\begin{gathered}
-|\delta|^{2} Y^{\prime \prime}(t)+P(t) Y(t)=\mu Y(t), \\
Y(t+2 \pi)=e^{i 2 \pi v(\beta)} Y(t),
\end{gathered}
$$

where $P(t)=\left(p_{i j}(t)\right), p_{i j}(t)=\sum_{k=1}^{\infty} v_{i j n_{k} \delta} e^{i n_{k} t}, v_{i j n_{k} \delta}=\left(v_{i j}(x), \frac{1}{\left|A_{n_{k} \delta}\right|} \sum_{\alpha \in A_{n_{k} \delta}} e^{i(\alpha \cdot x)}\right)$, $t=x \cdot \delta$. It means that $T(\gamma, \delta)$ is the infinite matrix $\left(T e^{i\left(l+n_{k}+v\right) t}, e^{i\left(l+n_{m}+v\right) t}\right)$, $k, m=1,2, \ldots$. 
To find the relation between the eigenvalues of $L(V)$ in a single resonance domain and the eigenvalues of the Sturm-Liouville operators defined by (55), we need the following theorems.

Theorem 4. Let $\gamma \in V_{\delta}\left(\rho^{\alpha_{1}}\right) \backslash E_{2}$ and $|\gamma| \sim \rho$. Then, for any eigenvalue $\eta_{j_{s}}(\gamma)$ of the matrix $C(\gamma, \delta)$ satisfying

$$
\left.\left|\eta_{j_{s}}-\right| h_{s}\right|^{2} \mid<M, \quad 1+(s-1) m \leq j_{s} \leq s m, \quad s=1,2, \ldots, a_{1}
$$

there exists an eigenvalue $\widetilde{\eta}_{k\left(j_{s}\right)}$ of the matrix $D(\gamma, \delta)$ such that

$$
\eta_{j_{s}}=\widetilde{\eta}_{k\left(j_{s}\right)}+O\left(\rho^{-\frac{3}{4} \alpha_{2}}\right)
$$

Proof. Let $\eta_{j_{s}}$ be an eigenvalue of the matrix $C(\gamma, \delta)$ satisfying (56) and $\theta_{j_{s}}=$ $\left(\theta_{j_{s}}^{1}, \theta_{j_{s}}^{2}, \ldots, \theta_{j_{s}}^{b_{1}}\right)_{m b_{1} \times 1}$ be the corresponding normalized eigenvector, $\left|\theta_{j_{s}}\right|=1$. Now, we consider the decomposition $C=A+B$ and the matrices $A, B$ which are defined in (37). Writing the binding formula (39) for $\eta_{j_{s}}$ and using (38), we get

$$
\left(\eta_{j_{s}}-\left|h_{\tau}\right|^{2}\right) \theta_{j_{s}}\left(h_{\tau, i}\right)=\theta_{j_{s}} \cdot B e_{i},
$$

$\tau=1,2, \ldots, b_{1}, \quad 1+(\tau-1) m \leq i \leq \tau m$.

For simplicity, we use the following notation in the sequel:

$$
\begin{gathered}
e_{\zeta, k}=e_{k} \quad \text { if } \quad 1+(\zeta-1) m \leq k \leq \zeta m, \quad \zeta=1, \ldots, b_{1}, \\
B e_{i} \cdot e_{k_{1}}=B e_{\tau, i} \cdot e_{\xi, k_{1}}=b\left(\tau, i, \xi, k_{1}\right) .
\end{gathered}
$$

Thus, substituting the orthogonal decomposition

$$
B e_{i}=B e_{\tau, i}=\sum_{\substack{\xi=1,2, \ldots, b_{1} \\ 1+(m-1) \xi \leq k_{1} \leq m \xi}} b\left(\tau, i, \xi, k_{1}\right) e_{\xi, k_{1}}
$$

into the formula (57), we get

$$
\begin{aligned}
\left(\eta_{j_{s}}-\left|h_{\tau}\right|^{2}\right) \theta_{j_{s}}\left(h_{\tau, i}\right) & =\theta_{j_{s}} \cdot \sum_{\substack{\xi=1,2, \ldots, b_{1} \\
1+(m-1) \xi \leq k_{1} \leq m \xi}} b\left(\tau, i, \xi, k_{1}\right) e_{\xi, k_{1}} \\
& =\sum_{\substack{\xi=1,2, \ldots, b_{1} \\
1+(m-1) \xi \leq k_{1} \leq m \xi}} b\left(\tau, i, \xi, k_{1}\right) \theta_{j_{s}} \cdot e_{\xi, k_{1}} \\
& =\sum_{\substack{\xi=1,2, \ldots, b_{1} \leq m \xi \\
1+(m-1) \xi \leq k_{1} \leq m \xi}} b\left(\tau, i, \xi, k_{1}\right) \theta_{j_{s}}\left(h_{\xi}, k_{1}\right) .
\end{aligned}
$$

It is clear that

which implies

$$
b\left(\tau, i, \xi, k_{1}\right)= \begin{cases}0 & \text { if } \xi=\tau, \\ v_{k_{1} i h_{\xi}-h_{\tau}} & \text { if } \xi \neq \tau\end{cases}
$$

$$
\sum_{\substack{\xi=1,2, \ldots, b_{1} \\
1+(m-1) \xi \leq k_{1} \leq m \xi}} b\left(\tau, i, \xi, k_{1}\right)=\sum \begin{gathered}
\xi=1,2, \ldots, b_{1} \\
v \\
k_{1} i h_{\xi}-h_{\tau}
\end{gathered} .
$$


Thus one has

$$
\begin{aligned}
& \left(\eta_{j_{s}}-\left|h_{\tau}\right|^{2}\right) \theta_{j_{s}}\left(h_{\tau}, i\right)=\sum \underset{v=1,2, \ldots, b_{1}}{v}{ }_{k_{1} i h_{\xi}-h_{\tau}} \theta_{j_{s}}\left(h_{\xi}, k_{1}\right) \\
& =\sum \begin{array}{c}
\xi=1,2, \ldots, a_{1} \\
v \\
k_{1} i h_{\xi}-h_{\tau}
\end{array} \theta_{j_{s}}\left(h_{\xi}, k_{1}\right) \\
& +\sum \begin{array}{c}
\xi=a_{1}+1, \ldots, b_{1} \\
v \\
k_{1} i h_{\xi}-h_{\tau}
\end{array} \theta_{j_{s}}\left(h_{\xi}, k_{1}\right) .
\end{aligned}
$$

Now, writing the equation $(58)$ for all $h_{\tau}, \tau=1,2, \ldots, a_{1}$, we get the system of linear algebraic equations:

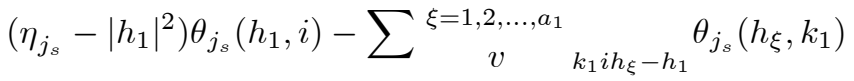

$$
\begin{aligned}
& =\sum \underset{v=a_{1}+1, \ldots, b_{1}}{v} \theta_{k_{1} i h_{\xi}-h_{1}} \theta_{j_{s}}\left(h_{\xi}, k_{1}\right) \\
& \left(\eta_{j_{s}}-\left|h_{2}\right|^{2}\right) \theta_{j_{s}}\left(h_{2}, i\right)-\sum \underset{v=1,2, \ldots, a_{1}}{v} k_{k_{1} i h_{\xi}-h_{2}} \theta_{j_{s}}\left(h_{\xi}, k_{1}\right)
\end{aligned}
$$

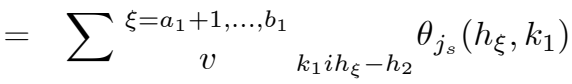

$$
\begin{aligned}
& \vdots \\
& \left(\eta_{j_{s}}-\left|h_{a_{1}}\right|^{2}\right) \theta_{j_{s}}\left(h_{a_{1}}, i\right)-\sum \underset{v}{\xi=1,2, \ldots, a_{1}}{ }_{k_{1} i h_{\xi}-h_{a_{1}}} \theta_{j_{s}}\left(h_{\xi}, k_{1}\right)
\end{aligned}
$$

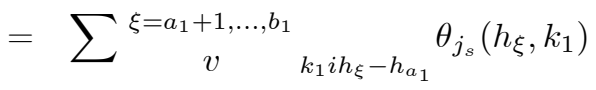

Using the binding formula (57), the relation (53), and $\|B\| \leq M$, for any $\tau=$ $1,2, \ldots, a_{1}$, we find

$$
\begin{aligned}
\left|\sum_{\substack{\xi=a_{1}+1, \ldots, b_{1} \\
k_{1}=1,2, \ldots, m, m \\
\xi \neq \tau}} v_{k_{1} i h_{\xi}-h_{\tau}} \theta_{j_{s}}\left(h_{\xi}, k_{1}\right)\right| & =\mid \sum_{\substack{\xi=a_{1}+1, \ldots, b_{1} \\
k_{1}=1,2, \ldots, m \\
\xi \neq \tau}} v_{k_{1} i h_{\xi}-h_{\tau}} \frac{\theta_{j_{s}} \cdot B e_{\xi, k_{1}}}{\left(\eta_{j_{s}}-\left|h_{\xi}\right|^{2}\right)} \\
& \leq \sum_{\substack{\xi=a_{1}+1, \ldots, b_{1} \\
k_{1}=1,2, \ldots, m \\
\xi \neq \tau}}\left|v_{k_{1} i h_{\xi}-h_{\tau}}\right| \frac{\theta_{j_{s}}|| B||\left|e_{\xi, k_{1}}\right|}{\left(\eta_{j_{s}}-\left|h_{\xi}\right|^{2}\right)} \\
& \leq 4 \rho^{-\alpha_{2}} M \sum_{\substack{\xi=a_{1}+1, \ldots, b_{1} \\
k_{1}=1,2, \ldots, m \\
\xi \neq \tau}}\left|v_{k_{1} i h_{\xi}-h_{\tau} \mid}\right| \\
& \leq 4 \rho^{-\alpha_{2}} M^{2} \\
& =O\left(\rho^{-\alpha_{2}}\right)
\end{aligned}
$$


and

$$
\begin{aligned}
\sum_{\substack{\tau=a_{1}+1, \ldots, b_{1} \\
i=1,2, \ldots, m}}\left|\theta_{j_{s}}\left(h_{\tau}, i\right)\right|^{2} & =\sum_{\substack{\tau=a_{1}+1, \ldots, b_{1} \\
i=1,2, \ldots, m}}\left|\frac{\theta_{j_{s}} \cdot B e_{\tau, i}}{\left(\eta_{j_{s}}-\left|h_{\tau}\right|^{2}\right)}\right|^{2} \\
& =\sum_{\substack{\tau=a_{1}+1, \ldots, b_{1} \\
i=1,2, \ldots, m}} \frac{\left|B \theta_{j_{s}} \cdot e_{\tau, i}\right|^{2}}{\left(\eta_{j_{s}}-\left|h_{\tau}\right|^{2}\right)^{2}} \\
& \leq 16 M^{2} \rho^{-2 \alpha_{2}} \\
& =O\left(\rho^{-2 \alpha_{2}}\right) .
\end{aligned}
$$

By 60 and 54 , (59) becomes

$$
\left[\theta_{j_{s}}^{1}, \theta_{j_{s}}^{2}, \ldots, \theta_{j_{s}}^{a_{1}}\right]^{t}=\left(D(\gamma, \delta)-\eta_{j_{s}} I\right)^{-1}\left[O\left(\rho^{-\alpha_{2}}\right), O\left(\rho^{-\alpha_{2}}\right), \ldots, O\left(\rho^{-\alpha_{2}}\right)\right]^{t} .
$$

By the Parseval's identity and 61, we get

$$
\begin{aligned}
\sum_{\substack{\tau=1,2, \ldots, a_{1} \\
i=1,2, \ldots, m}}\left|\theta_{j_{s}}\left(h_{\tau}, i\right)\right|^{2} & =\sum_{\substack{\tau=1,2, \ldots, b_{1} \\
i=1,2, \ldots, m}}\left|\theta_{j_{s}}\left(h_{\tau}, i\right)\right|^{2}-\sum_{\substack{\tau=a_{1}+1, \ldots, b_{1} \\
i=1,2, \ldots, m}}\left|\theta_{j_{s}}\left(h_{\tau}, i\right)\right|^{2} \\
& \geq 1-O\left(\rho^{-2 \alpha_{2}}\right) .
\end{aligned}
$$

Now, taking norm of both sides in 62 and using the above inequality we have

$$
\sqrt{1-O\left(\rho^{-2 \alpha_{2}}\right)}<\left(\sum_{\substack{\tau=1,2, \ldots, a_{1} \\ i=1,2, \ldots, m}}\left|\theta_{j_{s}}\left(h_{\tau}, i\right)\right|^{2}\right)^{\frac{1}{2}} \leq\left\|\left(D(\gamma, \delta)-\eta_{j_{s}} I\right)^{-1}\right\| O\left(\sqrt{a_{1}} \rho^{-\alpha_{2}}\right) .
$$

Thus

$$
\max \left|\eta_{j_{s}}-\widetilde{\eta}_{k\left(j_{s}\right)}\right|^{-1}>\frac{\sqrt{1-O\left(\rho^{-2 \alpha_{2}}\right)}}{\sqrt{a_{1}} \rho^{-\alpha_{2}}},
$$

or

$$
\min \left|\eta_{j_{s}}-\widetilde{\eta}_{k\left(j_{s}\right)}\right|=O\left(\sqrt{a_{1}} \rho^{-\alpha_{2}}\right)=O\left(\rho^{-\frac{3}{4} \alpha_{2}}\right),
$$

where the maximum (minimum) is taken over all $\widetilde{\eta}_{k\left(j_{s}\right)}, s=1,2, \ldots, a_{1}$. So the result follows.

Theorem 5. For any eigenvalue $\widetilde{\eta}_{\tau}$ of the matrix $D(\gamma, \delta)$, there exists an eigenvalue $\eta_{j_{s}(\tau)}$ of the matrix $C(\gamma, \delta)$ such that

$$
\eta_{j_{s}(\tau)}=\widetilde{\eta}_{\tau}+O\left(\rho^{-\frac{1}{2} \alpha_{2}}\right)
$$


Proof. Define the matrix $D^{\prime}=D^{\prime}(\gamma, \delta)$ by

$$
D^{\prime}=\left[\begin{array}{cccccccc}
\left|h_{1}\right|^{2} I-V_{0} & V_{h_{1}}-h_{2} & \cdots & V_{h_{1}-h_{a_{1}}} & 0 & 0 & \cdots & 0 \\
V_{h_{2}-h_{1}} & \left|h_{2}\right|^{2} I-V_{0} & \cdots & V_{h_{2}-h_{a_{1}}} & 0 & 0 & \cdots & 0 \\
\vdots & & & & & & & \\
V_{h_{a_{1}}-h_{1}} & V_{h_{a_{1}}-h_{2}} & \cdots & \left|h_{a_{1}}\right|^{2} I-V_{0} & 0 & 0 & \cdots & 0 \\
0 & 0 & \cdots & 0 & \left|h_{a_{1}+1}\right|^{2} I & 0 & \cdots & 0 \\
\vdots & \vdots & \cdots & \vdots & \vdots & \ddots & \cdots & 0 \\
0 & 0 & \cdots & 0 & 0 & 0 & \left|h_{b_{1}-1}\right|^{2} I & 0 \\
0 & 0 & \cdots & 0 & 0 & 0 & 0 & \left|h_{b_{1}}\right|^{2} I
\end{array}\right] \text { (63) }
$$

So that the spectrum of the matrix $D^{\prime}$ is

$$
\begin{aligned}
\operatorname{spec}\left(D^{\prime}\right) & =\operatorname{spec}(D(\gamma, \delta)) \bigcup\left\{\left|h_{a_{1}+1}\right|^{2},\left|h_{a_{1}+2}\right|^{2}, \ldots,\left|h_{b_{1}}\right|^{2}\right\} \\
& \equiv\left\{\widetilde{\eta}_{1}, \widetilde{\eta}_{2}, \ldots, \widetilde{\eta}_{m a_{1}},\left|h_{a_{1}+1}\right|^{2},\left|h_{a_{1}+2}\right|^{2}, \ldots,\left|h_{b_{1}}\right|^{2}\right\} .
\end{aligned}
$$

Let us denote by $\Upsilon_{\tau}=\left(\Upsilon_{\tau}^{1}, \Upsilon_{\tau}^{2}, \ldots, \Upsilon_{\tau}^{a_{1}}, 0, \ldots, 0\right)_{m b_{1} \times 1}, \Upsilon_{\tau}^{i}=\left(\Upsilon_{\tau}^{i 1}, \Upsilon_{\tau}^{i 2}, \ldots, \Upsilon_{\tau}^{i m}\right)_{m \times 1}$ the normalized eigenvector corresponding to the $\tau$-th eigenvalue of the matrix $D^{\prime}$, for $\tau=1,2, \ldots, m a_{1}$ and by $\left\{e_{k, i}\right\}_{i=1,2, \cdots, m}$ the eigenvector corresponding to the $k$-th eigenvalue $\left|h_{k}\right|^{2}$ of $D^{\prime}$, for $k=a_{1}+1, a_{1}+2, \ldots, b_{1}$.

Now, using $(62)$ from the previous theorem, we have

$$
\begin{aligned}
& \left(D^{\prime}-\eta_{j_{s}} I\right)\left[\theta_{j_{s}}^{1}, \theta_{j_{s}}^{2}, \ldots, \theta_{j_{s}}^{b_{1}}\right]^{t} \\
= & {\left[\left(D(\gamma, \delta)-\eta_{j_{s}} I\right)\left[\theta_{j_{s}}^{1}, \theta_{j_{s}}^{2}, \ldots, \theta_{j_{s}}^{a_{1}}\right]^{t},\left(\left|h_{a_{1}+1}\right|^{2}-\eta_{j_{s}}\right) \theta_{j_{s}}^{a_{1}+1}, \ldots,\left(\left|h_{b_{1}}\right|^{2}-\eta_{j_{s}}\right) \theta_{j_{s}}^{b_{1}}\right] } \\
= & {\left[O\left(\rho^{-\alpha_{2}}\right), \ldots, O\left(\rho^{-\alpha_{2}}\right),\left(\left|h_{a_{1}+1}\right|^{2}-\eta_{j_{s}}\right) \theta_{j_{s}}^{a_{1}+1}, \ldots,\left(\left|h_{b_{1}}\right|^{2}-\eta_{j_{s}}\right) \theta_{j_{s}}^{b_{1}}\right] . }
\end{aligned}
$$

Taking inner product of both sides of the last equality by $\Upsilon_{\tau}$ for $\tau=1,2, \ldots, m a_{1}$, using that $D^{\prime}$ is symmetric and $D^{\prime} \Upsilon_{\tau}=\widetilde{\eta}_{\tau} \Upsilon_{\tau}$ we have

$$
\left(\eta_{j_{s}(\tau)}-\widetilde{\eta}_{\tau}\right) \sum_{k=1}^{a_{1}} \theta_{j_{s}}^{k} \cdot \Upsilon_{\tau}^{k}=\sum_{k=1}^{a_{1}} O\left(\rho^{-\alpha_{2}}\right) \Upsilon_{\tau}^{k}
$$

For the right hand side of the equation (64) using the Cauchy-Schwarz inequality, we get

$$
\left|\sum_{k=1}^{a_{1}} O\left(\rho^{-\alpha_{2}}\right) \Upsilon_{\tau}^{k}\right| \leq \sqrt{\sum_{k=1}^{a_{1}} O\left(\rho^{-\alpha_{2}}\right)^{2}} \sqrt{\sum_{k=1}^{a_{1}}\left|\Upsilon_{\tau}^{k}\right|^{2}} \leq \sqrt{a_{1}\left(\rho^{-\alpha_{2}}\right)^{2}}=O\left(\sqrt{a_{1}} \rho^{-\alpha_{2}}\right),
$$

where $a_{1}=O\left(\rho^{\frac{1}{2} \alpha_{2}}\right)$. Thus, the equation 64 can be written as

$$
\left(\eta_{j_{s}(\tau)}-\widetilde{\eta}_{\tau}\right) \sum_{k=1}^{a_{1}} \theta_{j_{s}}^{k} \cdot \Upsilon_{\tau}^{k}=O\left(\rho^{-\frac{3}{4} \alpha_{2}}\right)
$$


In order to get the result, we need to show that for any $\tau=1,2, \ldots, m a_{1}$ there exists $\theta_{j_{s}(\tau)}$ such that

$$
\left|\sum_{k=1}^{a_{1}} \theta_{j_{s}(\tau)}^{k} \cdot \Upsilon_{\tau}^{k}\right|=\left|\theta_{j_{s}(\tau)} \cdot \Upsilon_{\tau}\right|>\sqrt{\frac{1-O\left(\rho^{-\frac{3}{2} \alpha_{2}}\right)}{m a_{1}}}>c_{18} \rho^{-\frac{1}{4} \alpha_{2}}
$$

For this, we consider the orthogonal decomposition $\Upsilon_{\tau}=\sum_{s=1}^{m b_{1}}\left(\Upsilon_{\tau} \cdot \theta_{j_{s}}\right) \theta_{j_{s}}$ and the Parseval's identity

$$
1=\sum_{s=1}^{m b_{1}}\left|\Upsilon_{\tau} \cdot \theta_{j_{s}}\right|^{2}=\sum_{s=1}^{m a_{1}}\left|\Upsilon_{\tau} \cdot \theta_{j_{s}}\right|^{2}+\sum_{s=m a_{1}+1}^{m b_{1}}\left|\Upsilon_{\tau} \cdot \theta_{j_{s}}\right|^{2} .
$$

First, let us show that

$$
\sum_{s=m a_{1}+1}^{m b_{1}}\left|\Upsilon_{\tau} \cdot \theta_{j_{s}}\right|^{2}=O\left(\rho^{-\frac{3}{2} \alpha_{2}}\right) .
$$

Using the decomposition $\Upsilon_{\tau}=\sum_{\substack{k=1,2 \ldots, a_{1} \\ i=1,2, \ldots, m}}\left(\Upsilon_{\tau} \cdot e_{k, i}\right) e_{k, i}$, the binding formula f57 for $C(\gamma, \delta)$ and $A$, the relation (53), and the Bessel's inequality we obtain the estimation

$$
\begin{aligned}
& \sum_{s=m a_{1}+1}^{m b_{1}}\left|\Upsilon_{\tau} \cdot \theta_{j_{s}}\right|^{2} \\
& =\sum_{s=m a_{1}+1}^{m b_{1}}\left|\left(\sum_{\substack{k=1,2, \ldots, a_{1} \\
i=1,2, \ldots, m}} \Upsilon_{\tau}^{k i} e_{k, i}\right) \cdot \theta_{j_{s}}\right|^{2} \\
& =\sum_{s=m a_{1}+1}^{m b_{1}}\left|\sum_{\substack{k=1,2, \ldots, a_{1} \\
i=1,2, \ldots, m}} \Upsilon_{\tau}^{k i}\left(e_{k, i} \cdot \theta_{j_{s}}\right)\right|^{2}=\sum_{s=m a_{1}+1}^{m b_{1}}\left|\sum_{\substack{k=1,2, \ldots, a_{1} \\
i=1,2, \ldots, m}} \Upsilon_{\tau}^{k i} \frac{\theta_{j_{s}} \cdot B e_{k, i}}{\left(\eta_{j_{s}}-\left|h_{k}\right|^{2}\right)}\right|^{2} \\
& \leq 16 \sum_{s=m a_{1}+1}^{m b_{1}} \rho^{-2 \alpha_{2}}\left(\sum_{\substack{k=1,2, \ldots, a_{1} \\
i=1,2, \ldots, m}}\left|\Upsilon_{\tau}^{k i}\right|\left|\theta_{j_{s}} \cdot B e_{k, i}\right|\right)^{2} \\
& \leq \sum_{s=m a_{1}+1}^{m b_{1}} 16\left|a_{1}\right| m \rho^{-2 \alpha_{2}}\left(\sum_{\substack{k=1,2, \ldots, a_{1} \\
i=1,2, \ldots, m}}\left|\Upsilon_{\tau}^{k i}\right|^{2}\left|\theta_{j_{s}} \cdot B e_{k, i}\right|^{2}\right) \\
& \leq 16 \rho^{-2 \alpha_{2}}\left|a_{1}\right| m \sum_{\substack{k=1,2, \ldots, a_{1} \\
i=1,2, \ldots, m}}\left|\Upsilon_{\tau}^{k i}\right|^{2} \sum_{s=m a_{1}+1}^{m b_{1}}\left|\theta_{j_{s}} B e_{k, i}\right|^{2}
\end{aligned}
$$




$$
\begin{aligned}
& \leq 16 \rho^{-2 \alpha_{2}}\left|a_{1}\right| m \sum_{\substack{k=1, \ldots, a_{1} \\
i=1,2, \ldots, m}}\left|\Upsilon_{\tau}^{k i}\right|^{2}\left|B e_{k, i}\right|^{2} \leq 16 \rho^{-2 \alpha_{2}}\left|a_{1}\right| m M^{2} \sum_{\substack{k=1,2, \ldots, a_{1} \\
i=1,2, \ldots, m}}\left|\Upsilon_{\tau}^{k i}\right|^{2} \\
& \leq 16\left|a_{1}\right| m \rho^{-2 \alpha_{2}} M^{2}=O\left(\rho^{-\frac{3}{2} \alpha_{2}}\right) .
\end{aligned}
$$

Therefore one has

$$
\sum_{s=1}^{m a_{1}}\left|\Upsilon_{\tau} \cdot \theta_{j_{s}}\right|^{2}=1-O\left(\rho^{-\frac{3}{2} \alpha_{2}}\right)
$$

from which it follows that there exists an eigenvector $\theta_{j_{s}(\tau)}$ such that 66 holds. Dividing both sides of 65 by 66 we get the result

$$
\eta_{j_{s}(\tau)}=\widetilde{\eta_{\tau}}+O\left(\rho^{-\frac{1}{2} \alpha_{2}}\right)
$$

Theorem 6. For every eigenvalue $\varsigma_{s}$ of the Sturm-Liouville operator $T(\gamma, \delta)$, there exists an eigenvalue $\widetilde{\varsigma_{s}}$ of the matrix $E(\gamma, \delta)$ such that

$$
\varsigma_{s}=\widetilde{\varsigma_{s}}+O\left(\rho^{-\frac{3}{4} \alpha_{2}}\right) .
$$

Proof. Decompose the infinite matrix $T(\gamma, \delta)$ as $T(\gamma, \delta)=\widetilde{A}+\widetilde{B}$ where the matrix $\widetilde{A}$ is defined by

$$
\widetilde{A}=\left[\begin{array}{ccc}
\left((l+v)^{2}|\delta|^{2}\right) I+V_{0} & \left((l-1+v)^{2}|\delta|^{2}\right) I+V_{0} & 0 \\
& \ddots & \left(\left(l-\frac{a_{1}}{2}+v\right)^{2}|\delta|^{2}\right) I+V_{0}
\end{array}\right]
$$

and $\widetilde{B}=T(\gamma, \delta)-\widetilde{A}$. Let $\varsigma_{s}$ be an eigenvalue of $T(\gamma, \delta)$, and $\Theta_{s}=\left(\Theta_{s}^{1}, \Theta_{s}^{2}, \Theta_{s}^{3}, \ldots\right)$, $\Theta_{s}^{\tau}=\left(\Theta_{s}^{\tau 1}, \ldots, \Theta_{s}^{\tau m}\right)$ be the corresponding normalized eigenvector, that is, $T \Theta_{s}=$ $\varsigma_{s} \Theta_{s} . \operatorname{span}\left\{e_{i}:(\tau-1) m+1 \leq i \leq \tau m\right\}$ is the eigenspace of the matrix $\tilde{A}$ which corresponds to the eigenvalue $\left|\left(\tau^{\prime}+v\right) \delta\right|^{2}$, where $\tau^{\prime}=l-\frac{\tau}{2}$ if $\tau$ is even, $\tau^{\prime}=l+\frac{\tau-1}{2}$ if $\tau$ is odd, for $\tau=1,2, \ldots$ and $\left\{e_{i}\right\}$ is the standard basis for $l_{2}$.

One can easily verify that

$$
\left(\varsigma_{s}-\left|\left(\tau^{\prime}+v\right) \delta\right|^{2}\right) \Theta_{s}^{\tau}=\Theta_{s} \cdot \widetilde{B} e_{\tau, i}
$$

where $e_{\tau, i} \equiv e_{i}$, if $(m-1) \tau+1 \leq i \leq m \tau$. to

Using the orthogonal decomposition $\widetilde{B} e_{\tau, i}=\sum_{j=1}^{m} \sum_{k=1}^{\infty}\left(\widetilde{B} e_{\tau, i} \cdot e_{k, j}\right) e_{k, j}, 69$ reduces

$$
\left(\varsigma_{s}-\left|\left(\tau^{\prime}+v\right) \delta\right|^{2}-\left|v_{i i 0}\right|^{2}\right) \Theta_{s}^{\tau i}=\sum_{j=1}^{m} \sum_{k=1}^{\infty}\left(\widetilde{B} e_{\tau, i} \cdot e_{k, j}\right) \Theta_{s}^{k j}
$$


and since $\widetilde{B} e_{\tau, i} \cdot e_{k, j}=v_{j i\left(n_{k}-n_{\tau}\right) \delta}$ for $k \neq \tau$,

$$
\left(\varsigma_{s}-\left(\tau^{\prime}+v\right) \delta^{2}\right) \Theta_{s}^{\tau i}-\sum_{j=1}^{m} \sum_{k=1}^{a_{1}} v_{j i\left(n_{k}-n_{\tau}\right) \delta} \Theta_{s}^{k j}=\sum_{j=1}^{m} \sum_{k=a_{1}+1}^{\infty} v_{j i\left(n_{k}-n_{\tau}\right) \delta} \Theta_{s}^{k j} .
$$

Now take any eigenvalue $\varsigma_{s}$ of $T(\gamma, \delta)$, satisfying $\left.\left|\varsigma_{s}-\right|\left(i^{\prime}+v\right) \delta\right|^{2}|<\sup | P(t) \mid$ for $s=1,2, \ldots, \frac{m a_{1}}{2}$, where $i^{\prime}=l-\frac{s}{2}$ if $s$ is even, $i^{\prime}=l+\frac{s-1}{2}$ if $s$ is odd. The relations $\gamma \in V_{\delta}\left(\rho^{\alpha_{1}}\right)\left(\delta \neq e_{i}\right)$ and $\gamma=\beta+(l+v) \delta, \beta \cdot \delta=0$ imply

$$
\left.|2 \gamma \cdot \delta+| \delta\right|^{2}|=|(l+v)|\delta|^{2}+|\delta|^{2}\left|<\rho^{\alpha_{1}}, \quad\right| l \mid<c_{19} \rho^{\alpha_{1}} .
$$

Therefore, using the definition of $i^{\prime}$ and $\tau^{\prime}$, we have

$$
\left|\left(i^{\prime}+v\right) \delta\right|<\frac{\left|a_{1} \delta\right|}{4}+c_{20} \rho^{\alpha_{1}}
$$

for $s=1,2, \ldots \frac{a_{1}}{2}$ and

$$
\left|\left(\tau^{\prime}+v\right) \delta\right|>\frac{\left|a_{1} \delta\right|}{2}-c_{21} \rho^{\alpha_{1}}
$$

for $\tau>a_{1}$. Since $\left|a_{1}\right|>c_{22} \rho^{\frac{\alpha_{2}}{2}}$ and $\alpha_{2}>2 \alpha_{1}$, we have

$$
\left.||\left(i^{\prime}+v\right) \delta\right|^{2}-\left|\left(\tau^{\prime}+v\right) \delta\right|^{2} \mid>c_{23} \rho^{\alpha_{2}}
$$

for $s \leq \frac{a_{1}}{2}, \tau>a_{1}$, which implies

$$
\left|\varsigma_{s}-\right|\left(\tau^{\prime}+v\right)\left|\delta^{2}\right|=|| \varsigma_{s}-\left.||\left(i^{\prime}+v\right) \delta\right|^{2}|-|\left|\left(\tau^{\prime}+v\right)\right| \delta^{2}|-|\left|\left(i^{\prime}+v\right) \delta\right|^{2}||>c_{24} \rho^{\alpha_{2}},
$$

for $s=1,2, \ldots \frac{a_{1}}{2}, \tau>a_{1}$.

Since $\widetilde{B}$ corresponds to the operator $P: Y \rightarrow P(t) Y$ in $L_{2}^{m}[0,2 \pi]$, which has norm $\sup |P(t)| \leq M$. Using this, equation (69) and $(72)$, we have for the right hand side of 70 that

$$
\begin{gathered}
\left|\sum_{j=1}^{m} \sum_{k=a_{1}+1}^{\infty} v_{i j\left(n_{k}-n_{\tau}\right) \delta} \Theta_{s}^{k j}\right| \leqslant \sum_{j=1}^{m} \sum_{k=a_{1}+1}^{\infty}\left|v_{i j\left(n_{k}-n_{\tau}\right) \delta}\right|\left|\frac{\Theta_{s} \cdot \widetilde{B} e_{k j}}{\varsigma_{s}-\left|\left(k^{\prime}+v\right) \delta\right|^{2}}\right| \\
\leq \sum_{j=1}^{m} \sum_{k=a_{1}+1}^{\infty}\left|v_{i j\left(n_{k}-n_{\tau}\right) \delta \mid} \frac{\left\|\Theta_{s}\right\|\left\|\widetilde { B } \left|\left\|\mid e_{k j}\right\|\right.\right.}{\left.\left|\varsigma_{s}-\right|\left(k^{\prime}+v\right) \delta\right|^{2} \mid} \leq M \rho^{-\alpha_{2}} \sum_{j=1}^{m} \sum_{k=a_{1}+1}^{\infty}\right| v_{i j\left(n_{k}-n_{\tau}\right) \delta} \mid \\
\leq c_{25} \rho^{-\alpha_{2}}
\end{gathered}
$$

Therefore writing the equation 70 for all $\tau=1,2, \ldots, a_{1}$, and using 73 we get the following system

$$
\left(E(\gamma, \delta)-\varsigma_{s} I\right)\left[\Theta_{s}^{1}, \Theta_{s}^{2}, \ldots, \Theta_{s}^{a_{1}}\right]=\left[O\left(\rho^{-\alpha_{2}}\right), O\left(\rho^{-\alpha_{2}}\right), \ldots, O\left(\rho^{-\alpha_{2}}\right)\right],
$$


where $I$ is an $m a_{1} \times m a_{1}$ identity matrix. Using $\Theta_{s}=\sum_{\tau=1}^{\infty} \Theta_{s}^{\tau} e_{\tau, i}$, the formula 69 and the inequality (72), we have

$$
\sum_{\tau=a_{1}+1}^{\infty}\left|\Theta_{s}^{\tau}\right|^{2}=\sum_{\tau=a_{1}+1}^{\infty}\left|\frac{\Theta_{s} \cdot \widetilde{B} e_{\tau, i}}{\varsigma_{s}-\left|\left(\tau^{\prime}+v\right) \delta\right|^{2}}\right|^{2}=O\left(\rho^{-2 \alpha_{2}}\right)
$$

and thus

$$
\sum_{\tau=1}^{a_{1}}\left|\Theta_{s}^{\tau}\right|^{2}=1-O\left(\rho^{-2 \alpha_{2}}\right) .
$$

Multiplying both sides of 74 by $\left(E(\gamma, \delta)-\varsigma_{s} I\right)^{-1}$,

$$
\left[\Theta_{s}^{1}, \Theta_{s}^{2}, \ldots, \Theta_{s}^{a_{1}}\right]=\left(E(\gamma, \delta)-\varsigma_{s} I\right)^{-1}\left[O\left(\rho^{-\alpha_{2}}\right), \ldots, O\left(\rho^{-\alpha_{2}}\right)\right],
$$

then taking norm of both sides and using $(75)$, we get

or

$$
\sqrt{\frac{1-O\left(\rho^{-2 \alpha_{2}}\right)}{m}}=\left\|\left(E(\gamma, \delta)-\varsigma_{s} I\right)^{-1}\right\| O\left(\sqrt{a_{1}} \rho^{-\alpha_{2}}\right)
$$

$$
\min _{\tau}\left|\varsigma_{s}-\widetilde{\varsigma}_{\tau}\right|=\frac{O\left(\sqrt{a_{1}} \rho^{-\alpha_{2}}\right) \cdot \sqrt{m}}{\sqrt{1-O\left(\rho^{-2 \alpha_{2}}\right)}}=O\left(\rho^{-\frac{3}{4} \alpha_{2}}\right)
$$

where the minimum is taken over all eigenvalues $\widetilde{\varsigma}_{\tau}$ of the matrix $E(\gamma, \delta)$. Thus, the result follows.

Theorem 7. (Main result) For every $\beta \in H_{\delta},|\beta| \sim \rho$ and for every eigenvalue $\varsigma_{s}(v(\beta))$ of the Sturm-Liouville operator $T(\gamma, \delta)$, there is an eigenvalue $\Lambda_{N}$ of the operator $L(V)$ satisfying

$$
\Lambda_{N}=|\beta|^{2}+\varsigma_{s}+O\left(\rho^{-\frac{1}{2} \alpha_{2}}\right) .
$$

Proof. From Theorem 6 and the definition of $E(\gamma, \delta)$, there exists an eigenvalue $\widetilde{\eta}_{\tau(s)}$ of the matrix $D(\gamma, \delta)$, where $\gamma$ has a decomposition $\gamma=\beta+(\tau+v(\beta)) \delta$, satisfying $\widetilde{\eta}_{\tau(s)}=|\beta|^{2}+\varsigma_{s}+O\left(\rho^{-\frac{3}{4} \alpha_{2}}\right)$. Therefore, the result follows from Theorem 5 and Theorem 2

\section{REFERENCES}

[1] Atılgan, Ş., Karakılıç, S. and Veliev, O. A., Asymptotic Formulas for the Eigenvalues of the Schrödinger Operator, Turk J Math., 26 (2002) 215-227.

[2] Berezin, F. A. and Shubin, M. A., The Schrödinger Equation, Kluwer Academic Publishers, Dordrecht, 1991.

[3] Coṣkan, D. and Karakılıç, S., High energy asymptotics for eigenvalues of the Schrödinger operator with a matrix potential, Mathematical Communications, 16(2) (2011).

[4] Feldman, J., Knoerrer, H. and Trubowitz, E., The Perturbatively Stable Spectrum of the Periodic Schrödinger Operator, Invent. Math., 100 (1990) 259-300.

[5] Feldman, J., Knoerrer, H. and E. Trubowitz, The Perturbatively Unstable Spectrum of the Periodic Schrödinger Operator, Comment. Math. Helvetica, 66(1991) 557-579.

[6] Friedlanger, L., On the Spectrum for the Periodic Problem for the Schrödinger Operator, Communications in Partial Differential Equations, 15(1990) 1631-1647. 
[7] Hald, O. H. and McLaughlin, J.R., Inverse Nodal Problems: Finding the Potential from Nodal Lines, Memoirs of AMS, 572, 119 (1996) 0075-9266.

[8] Karakılıç, S. and Akduman, S., Eigenvalue Asymptotics for the Schrödinger Operator with a Matrix Potential in a Single Resonance Domain, Filomat, 29(1) (2015) 21-38.

[9] Karakılı̧, S., Atılgan, Ş. and Veliev, O. A., Asymptotic Formulas for the Eigenvalues of the Schrödinger Operator with Dirichlet and Neumann Boundary Conditions, Reports on Mathematical Physics (ROMP), 55(2) (2005) 221-239.

[10] Karakılı̧, S., Veliev, O. A. and Atılgan, Ş., Asymptotic Formulas for the Resonance Eigenvalues of the Schrödinger Operator, Turkish Journal of Mathematics, 29(4) (2005) 323-347.

[11] Karpeshina, Y., Perturbation Theory for the Schrödinger Operator with a non-smooth Periodic Potential, Math. USSR-Sb, 71 (1992) 701-123.

[12] Karpeshina, Y., Perturbation series for the Schrödinger Operator with a Periodic Potential near Planes of Diffraction, Communication in Analysis and Geometry, 4(3) (1996) 339-413.

[13] Karpeshina, Y., On the Spectral Properties of Periodic Polyharmonic Matrix Operators, Indian Acad. Sci. (Math. Sci.), 112(1) (2002) 117-130.

[14] Kato, T., Perturbation Theory for Linear Operators, Springer Berlin, 1980.

[15] Reed, M. and Simon, B., Methods of Modern Mathematical Physics, 3rd ed., New York, San Francisco, London: Academic Press, vol. IV 1987.

[16] Naimark, M. A., Dawson, E. R. and Everitt, W. N., Linear Differential Operators, Part I, Elementary Theory of Linear Differential Operators, with additional material by the author, Frederick Ungar Publ., Co., New York, 196-6 1967.

[17] Veliev, O. A., On the spectrum of the Schrödinger operator with periodic potential, Dokl. Akad. Nauk SSSR., Vol. 268, No. 6 (1983).

[18] Veliev, O. A., Asymptotic Formulas for the Eigenvalues of the Periodic Schrödinger Operator and the Bethe-Sommerfeld Conjecture, Functsional Anal. i Prilozhen, 21(2) (1987) 1-15.

[19] Veliev, O. A., The Spectrum of Multidimensional Periodic Operators, Teor. Functional Anal. i Prilozhen, 49 (1988) 17-34.

[20] Veliev, O. A., Asymptotic Formulas for the Bloch Eigenvalues Near Planes of Diffraction, Reports on Mathematical Physics(ROMP), 58(3) (2006) 445-464.

[21] Veliev, O. A., Perturbation Theory for the Periodic Multidimensional Schrödinger Operator and the Bethe-Sommerfeld Conjecture, International Journal of Contemporary Mathematical Sciences, 2(2) (2007) 19-87.

[22] Veliev, O. A., Multidimensional periodic Schrödinger operator: Perturbation theory and applications.,Springer, Vol. 2632015.

Current address: Sedef Karakılıç: Dokuz Eylül University, İzmir Turkey.

E-mail address: sedef.erim@deu.edu.tr

ORCID Address: http://orcid.org/0000-0002-0407-0271

Current address: Setenay Akduman: İzmir Demokrasi University, İzmir Turkey.

E-mail address: setenay.akduman@idu.edu.tr

ORCID Address: http://orcid.org/0000-0003-2492-3734

Current address: Didem Coşkan: Dokuz Eylül University, İzmir Turkey.

E-mail address: coskan.didem@gmail.com

ORCID Address: http://orcid.org/0000-0003-2358-198X 